\title{
CpG-ODN Induces a Dose-Dependent Enrichment of Immunological Niches in the Spleen and Lungs of Neonatal Chicks That Correlates with the Protective Immunity against Escherichia coli
}

\author{
Thushari Gunawardana, ${ }^{1}$ Khawaja Ashfaque Ahmed $\left(\mathbb{D},{ }^{1}\right.$ Kalhari Goonewardene, \\ Shelly Popowich, ${ }^{1}$ Shanika Kurukulasuriya, ${ }^{1}$ Ruwani Karunarathana, ${ }^{1}$ \\ Lisanework E. Ayalew, ${ }^{1}$ Ashish Gupta, ${ }^{1}$ Betty Lockerbie, ${ }^{1}$ Marianna Foldvari, ${ }^{2}$ \\ Suresh K. Tikoo $\mathbb{D}^{3}{ }^{3}$ Philip Willson, ${ }^{4}$ and Susantha Gomis $\mathbb{D}^{1}$ \\ ${ }^{1}$ Department of Veterinary Pathology, Western College of Veterinary Medicine, University of Saskatchewan, Saskatoon, SK, Canada \\ S7N $5 B 4$ \\ ${ }^{2}$ School of Pharmacy, University of Waterloo, 200 University Avenue West, Waterloo, ON, Canada N2L 3G1 \\ ${ }^{3}$ Vaccinology and Immunotherapy, School of Public Health, University of Saskatchewan, Saskatoon, SK, Canada S7N 5E3 \\ ${ }^{4}$ Canadian Centre for Health and Safety in Agriculture, University of Saskatchewan, Saskatoon, SK, Canada S7N 5E5
}

Correspondence should be addressed to Khawaja Ashfaque Ahmed; kaa201@mail.usask.ca and Susantha Gomis; smg127@mail.usask.ca

Received 11 July 2019; Accepted 5 December 2019; Published 13 January 2020

Academic Editor: Senthamil R. Selvan

Copyright (C) 2020 Thushari Gunawardana et al. This is an open access article distributed under the Creative Commons Attribution License, which permits unrestricted use, distribution, and reproduction in any medium, provided the original work is properly cited.

Immunoprotective function of oligodeoxynucleotides containing $\mathrm{CpG}$ motifs (CpG-ODN) has been demonstrated in neonatal chickens against common bacterial pathogens such as E.coli and Salmonella sp. Our recent study reported that CpG-ODN administration enriches immune compartments in neonatal chicks. However, a causal relationship between CpG-ODN-induced immune enrichment and protective mechanisms remains unestablished. In this study, we investigated in ovo administered CpGODN-mediated immune cell recruitment in the immunological niches in lymphoid (spleen) and nonlymphoid (lungs) organs using various doses of $\mathrm{CpG}-\mathrm{ODN}$ and examined whether the immunological profiles have any correlation with immunoprotection against E.coli infection. Eighteen-day-old embryonated eggs were injected with either 5, 10, 25, and 50 $\mu \mathrm{g}$ of CpG-ODN or saline ( $n=\sim 40$ per group). On the day of hatch ( $72 \mathrm{hr}$ after CpG-ODN treatment), we collected the spleen and lungs ( $n=3-4$ per group) and examined the recruitment of macrophages/monocytes, their expression of MHCII and CD40, and the number of $\mathrm{CD}^{+}$and $\mathrm{CD}^{+}$T-cell subsets in the immunological niches in the spleen and lungs using flow cytometry. We observed the dose-dependent recruitment of immune cells, wherein $25 \mu \mathrm{g}$ and $50 \mu \mathrm{g}$ of CpG-ODN induced significant enrichment of immunological niches in both the spleen and the lungs. Four days after the CpG-ODN treatment (1-day after hatch), chicks were challenged with a virulent strain of E. coli $\left(1 \times 10^{4}\right.$ or $1 \times 10^{5} \mathrm{cfu}$, subcutaneously). Clinical outcome and mortality were monitored for 8 days postchallenge. We found that both $25 \mu \mathrm{g}$ and $50 \mu \mathrm{g}$ of CpG-ODN provided significant protection and reduced clinical scores compared to saline controls against $E$. coli infection. Overall, the present study revealed that $\mathrm{CpG}-\mathrm{ODNs}$ orchestrate immunological niches in neonatal chickens in a dose-dependent manner that resulted in differential protection against $E$. coli infection, thus supporting a cause and effect relationship between CpG-ODN-induced immune enrichment and the antibacterial immunity. 


\section{Introduction}

Infectious diseases of neonatal poultry are common due to the immaturity of the immune system or inadequate sensitization of the immune system to antigens [1]. During the first week of a bird's life, high mortality associated with bacterial infections, Escherichia coli septicemia in particular [2], has devastating impacts on poultry production [3]. Antimicrobials are effective in controlling bacterial diseases, and thus, the prophylactic use of antimicrobials is a common practice in the poultry industry [4]. However, these prophylactic use of antibiotics in the poultry industry may lead to antibiotic residues in poultry products $[5,6]$ and the emergence of antibiotic-resistant strains of bacteria [4, 7]. Hence, reduction of antimicrobial use is a priority of the poultry industry. Since the use of categoryl antibiotics has been discontinued since 2014 [8], the poultry industry needs suitable alternatives to antibiotics for controlling diseases in neonatal chickens $[9,10]$.

Innate immunity is the first line of defense against infectious agents. The host needs to identify an invading pathogen to mount a rapid immune response. The cells of the innate immune system rely on a set of pattern recognition receptors (PRR), which can detect specific molecular structures present in the pathogens known as pathogen-associated molecular patterns (PAMPs) [11]. The innate immune cells, like dendritic cells (DC), are activated following the interaction of PRR of DC with PAMP of a pathogen. Such PRR-PAMP interaction initiates cell signaling that mounts immune responses, eventually leading to the development of adaptive immunity against the invading pathogen. Toll-like receptors (TLRs) are the main PRR, which are important in the induction of innate immunity [12]. The components of pathogens such as lipopeptides, glycerophosphatidylinositol, lipopolysaccharides (LPS), microbial nucleic acids (dsRNA, ssRNA, and unmethylated CpG DNA), and microbial proteins (flagellin, profilin) are some of the well-known TLR ligands (PAMPs). Many potential TLR agonists have been suggested as immune modulators by different studies [13]. Synthetic CpG-ODNs are recognized by TLR-9 and TLR-21 in mammals and avian, respectively [14-18]. CpG-ODNs initiate immune responses in mice [19], fish [20], cattle and sheep [21], human [22-24], and chickens [25, 26]. CpG-ODNs are safe immunoprotective agents, and the Food and Drug Administration has approved its use in humans [27].

In quest of an alternative to antimicrobial agents against bacterial infections, our laboratory pioneered the use of CpG-ODN alone as an immune protective agent against $E$. coli infection in chickens $[28,29]$. We demonstrated that CpG-ODN administered through various routes protects chickens against E. coli $[4,26]$ and Salmonella Typhimurium infection $[29,30]$. The protective effect of CpG-ODN has also reported against Salmonella Enteritidis infection by other studies [31, 32]. Moreover, CpG-ODN formulation with nanoparticles further improved its immunoprotective action $[30,33,34]$. Several studies have also reported improved immune responses of nanoparticle formulated CpG-ODN $[17,18]$ and demonstrated enhanced expression of cytokines and chemokines following CpG-ODN administration in chickens $[34,35]$. The previous study showed that the resolution of Salmonella Typhimurium infection strongly correlated with proinflammatory cytokine expression in chickens [36]. Despite recent advances, the immunoprotective mechanism(s) of CpG-ODN alone against bacterial infections remained poorly understood.

Proinflammatory cytokines stimulate the secretion of chemokines and expression of cell-surface leukocyte adhesion molecules and promote the rapid recruitment of immune cells in the inflammatory area [37-39]. We found that the intrapulmonary delivery of CpG-ODN initiated the infiltration of inflammatory cells in the pulmonary parenchyma [4]. We recently found that in ovo administration of CpG-ODN enriches various immune compartments in neonatal chicks [40]. We hypothesize that CpG-ODNmediated protection may be through the regulation of immunological niches in neonatal chickens. Thus, the objective of this study was to evaluate the influx of macrophages and $\mathrm{CD}^{+}$and $\mathrm{CD}^{+}{ }^{+} \mathrm{T}$-cell subsets in the immunological niches such as the spleen and lung in chickens and investigate if immune profiles correlate with the CpG-ODN-induced protection against $E$. coli infection.

\section{Materials and Methods}

2.1. Experimental Chickens. All animal experiments were approved by the University of Saskatchewan's Animal Research Ethics Board and adhered to the Canadian Council on Animal Care guidelines for humane animal use. Fertilized hatching eggs were obtained from a commercial broiler breeder operation in Saskatchewan, Canada. Eggs were incubated at the Animal Care Unit (ACU) at the Western College of Veterinary Medicine, University of Saskatchewan. Following in ovo injections, eggs were transferred to the hatcher in separate groups. No significant differences were seen in fertility and hatchability due to in ovo injections between groups. Groups of hatched chicks were tagged for group identification and allocated into an animal isolation rooms at the ACU. Water and commercial broiler rations with no antibiotics were provided ad libitum to all groups in the same manner. Each room was ventilated with filtered, nonrecirculated air at a rate of 10-12 changes/hr. Air pressure differentials and strict sanitation were maintained in this isolation facility.

2.2. Synthetic $C p G-O D N$. The sequence of CpG-ODN (class B CpG 2007) used was $5^{\prime}$-TCGTCGTTGTCGTTTT GTCGTT-3'. ODNs were produced with a phosphorothioate backbone (Operon Biotechnologies, Inc. Huntsville, AL).

2.3. Delivery of CPG-ODN by In Ovo Route. Embryonated eggs (incubated for 18 days) received either 50, 25, 10, or $5 \mu \mathrm{g}$ of $\mathrm{CpG}-\mathrm{ODN}$ diluted in sterile pyrogen-free saline, in a total of $100 \mu \mathrm{l} / \mathrm{egg}$ or $100 \mu \mathrm{l}$ of sterile saline $(n=\sim 40$ /group). Injections were administered by the in ovo route into the amniotic cavity through the air cell side of the egg using a 22-gauge, 1-inch hypodermic needle. The volume of the injection and the length of the needle were selected to 
simulate the standard in ovo injection technology used in the poultry industry. Following in ovo injection, the injection sites of eggs were covered with melted paraffin applied with a wooden applicator and transferred to the hatcher until hatch.

\subsection{Tissue Sample Collection and Flow Cytometry. Three or} four embryos from each group were humanely euthanized at $72 \mathrm{hrs}$ post in ovo injections by cervical dislocation and necropsied for tissue collection. Spleen and lung tissues were collected into $1.5 \mathrm{ml}$ microcentrifuge tubes. Cell preparation and antibody staining for flow cytometry were done as previously described with some modifications [41, 42]. Briefly, each spleen was gently pushed through a metal strainer by manual pressure to obtain a single cell suspension with $\sim 10 \mathrm{ml}$ of phosphate-buffered saline (PBS) and collected to a $15 \mathrm{ml}$ centrifuge tube. For lung, each tissue was manually dissected and incubated with $\sim 1 \mathrm{ml}$ of collagenase type 4 (Sigma-Aldrich, St. Louis, Missouri, USA) $(1 \mathrm{mg} / \mathrm{ml})$ dissolved in Dulbecco's modified Eagle medium (DMEM) for 30 minutes in $37^{\circ} \mathrm{C}$; after incubation, these tissues were filtered through a metal strainer to obtain a single cell suspension and washed twice with PBS. Spleen and lung cells were then incubated with RBC lysis buffer to lyse red blood cells. Following three washes with wash buffer (PBS containing $2 \%$ fetal bovine serum and $0.1 \%$ sodium azide), cells were stained with appropriate antibodies.

The cell populations of the spleen and lungs collected at $72 \mathrm{hrs}$ post in ovo injections from each group $(n=3-4)$ were stained for the presence of antigen-presenting cells (APCs), $\mathrm{CD}^{+}$and $\mathrm{CD}^{+}$T-cell subsets. For detecting antigenpresenting cells (APCs) and their expression of cellular markers, such as CD40 and MHCII, spleen and lung cells $\left(\sim 5 \times 10^{5}\right.$ cells $)$ were incubated with mouse anti-chicken CD40 primary antibody (clone AV79; Bio-Rad, Raleigh, $\mathrm{NC}$, USA) at $4^{\circ} \mathrm{C}$ for $30 \mathrm{~min}$ followed by three washing steps before incubating with goat anti-mouse IgG-PerCP/Cy5.5 secondary antibody at $4^{\circ} \mathrm{C}$ for $30 \mathrm{~min}$. After three washes, the cells were stained with mouse anti-chicken monocyte/macrophage-PE (clone: KUL01; Southern Biotechnology, Birmingham, Ala, USA) and mouse anti-chicken MHCIIAF488 (clone: 2G11; Southern Biotechnology, Birmingham, Ala, USA) antibody together at $4^{\circ} \mathrm{C}$ for $30 \mathrm{~min}$. Subsequently, the cells were washed three times and suspended in $\sim 300 \mu \mathrm{l}$ flow cytometric buffer for the analysis. Another set of spleen and lung cells $\left(\sim 5 \times 10^{5}\right.$ cells $)$ were incubated with antichicken CD8-FITC (clone: EP72; Southern Biotechnology, Birmingham, Ala, USA) and CD4-PE (clone: CT-4; Southern Biotechnology, Birmingham, Ala, USA) together at $4^{\circ} \mathrm{C}$ for 30 min to determine $\mathrm{CD} 4^{+}$and $\mathrm{CD} 8^{+} \mathrm{T}$-cells. Following three washes, these cells were also suspended in $\sim 300 \mu \mathrm{l}$ flow cytometric buffer (PBS containing 2\% fetal bovine serum and $0.1 \%$ sodium azide) in flow cytometry tubes and processed for flow cytometric analysis. In flow cytometry, cells were gated based on forward vs. side scatter plot. Flow cytometry data were acquired by Epics XL (Beckman Coulter) and FACSCalibur (BD Bioscience), and data were analyzed with FlowJo software (Tree Star).
2.5. Bacteria. For the challenge, a field isolate of E. coli from a turkey with septicemia was used as previously described $[26,28]$, which was stored at $-80^{\circ} \mathrm{C}$ in $50 \%$ brain-heart infusion broth (BHI; Difco, Detroit, MI) supplemented with 25\% (w/v) glycerol (VWR Scientific, Inc., Montreal, Quebec). This E. coli strain was serogroup O2, nonhemolytic, with a K1 capsule and Type 1 pili, produced aerobactin and serum resistant. First, bacteria were cultured on Columbia sheep blood agar plates for 18-24hrs at $37^{\circ} \mathrm{C}$. Then, one colony was added to $100 \mathrm{ml}$ of Luria broth in a $250 \mathrm{ml}$ Erlenmeyer flask. The culture was grown at $37^{\circ} \mathrm{C}$ for $16-18 \mathrm{hrs}$ with shaking at $150 \mathrm{rpm}$, and optical density (OD) was measured at $600 \mathrm{~nm}$ wavelength using a spectrophotometer. This stationary phase culture contained approximately $1 \times 10^{9}$ colony-forming units (cfu) of bacteria per ml. Finally, cultures were diluted in sterile saline so the concentration of bacteria required for the challenge $\left(1 \times 10^{5}\right.$ or $1 \times 10^{4} \mathrm{cfu} / \mathrm{in} 250 \mu \mathrm{l}$ bird $)$ was obtained. Viable bacterial counts were determined by counting the number of colonies following plating serial dilutions of the challenge doses in duplicate on Columbia sheep blood agar plates and incubating for 18-24hrs at $37^{\circ} \mathrm{C}$.

2.6. E. coli Challenge Experiment. All the remaining birds (bird numbers per group; CpG-ODN $5 \mu \mathrm{g}: n=37$; CpGODN $10 \mu \mathrm{g}: n=33$; CpG-ODN $25 \mu \mathrm{g}: n=34$; CpG-ODN $50 \mu \mathrm{g}: n=30$, saline: $n=35)$ received either $1 \times 10^{5}(50 \%$ of birds in each group) or $1 \times 10^{4}$ (50\% of birds in each group) cfu of stationary phase E. coli, in a total volume of $250 \mu \mathrm{l}$ per bird, by subcutaneous injection in the neck one day posthatch. E. coli septicemia with airsacculitis, pericarditis, or perihepatitis develops in $60 \%-90 \%$ of birds that are not protected by treatment intervention, in this model. Clinical signs, pathology, bacterial isolations from the air sacs, and mortality were observed and recorded every day for 8 days following challenge with E. coli. Birds were evaluated three times daily at the critical stage, which is the first four days postchallenge, then twice daily for 7 days postchallenge. Birds were observed for clinical signs, and each individual was assigned a daily clinical score: $0=$ normal; $0.5=$ slightly abnormal appearance, slow to move; $1=$ depressed, reluctant to move; 1.5 = reluctant to move, may take a drink and peck some; $2=$ unable to stand or reach food or water; and $3=$ found dead. Birds that received a clinical score of 2 were humanely euthanatized by cervical dislocation. Chicks that were found dead or euthanatized were necropsied, and bacterial swabs were taken from air sacs immediately. On day 8 , after the E. coli challenge, all remaining birds were euthanatized by cervical dislocation. Bacterial swabs were taken from the air sacs and cultured on Columbia sheep blood agar using a typical method of streaking on four quadrants of the plate of the medium. A semiquantitative estimate of E. coli isolation was conducted on Columbia sheep blood agar. Growth on these plates were recorded on a scale from 0 to $4+$, where $0=$ no growth; $1+=$ growth of bacteria on the area $1 ; 2+=$ growth of the bacteria on areas 1 and $2 ; 3+=$ growth of bacteria on areas 1,2 , and 3 ; and $4+=$ growth of bacteria 
on areas 1, 2, 3, and 4. All lesions found in dead or euthanized chicks were recorded.

2.7. Statistical Analysis. Prism 5.0, GraphPad Software Inc., San Diego, CA, was used to analyze and graph survival trends, cumulative clinical scores (CCSs), bacterial percentages, and cell populations from flow cytometry analysis, with a significance level of $P<0.05$. The survival patterns and median survival times were compared using the log-rank test and the chi-square statistic. The reduction of the relative risk of mortality of groups of birds was calculated using Microsoft excel. The clinical score for each bird in the respective group was summed every day for 8-day observation period to calculate the CCS, and the significance of differences among groups was tested with the use of Kruskal-Wallis nonparametric analysis of variance. For testing significant differences in the means of immune cell numbers and their maturation marker expression between groups, ANOVA testing was done. Dunnett's test was used as a post hoc test following ANOVA to assess for significant differences between each treatment group compared to the saline control group. For analyzing the difference in $\mathrm{CD}^{+}$and $\mathrm{CD}^{+}{ }^{+}$-cells between groups, we used two-way ANOVA followed with the Bonferroni posttest and the Student T-test with Welch's correction for unequal variance was used, with a significant difference of $P<0.05$.

\section{Results}

3.1. Flow Cytometry. Flow cytometry analysis of the lungs and at $72 \mathrm{hrs}$ post in ovo injections (on the day of hatch) showed a significant influence of CpG-ODN on immune cell components of birds. Results demonstrated a dosedependent influence of CpG-ODN affecting cell population percentages and maturation marker expression levels.

\subsection{Immune Cell Profiling of the Lung}

3.2.1. Antigen-Presenting Cells in the Lung. CpG-ODN showed a strong effect on MHCII-expressing APC cell population (monocyte/macrophages) $72 \mathrm{hrs}$ post in ovo injections. The groups that received $25 \mu \mathrm{g}$ or $50 \mu \mathrm{g}$ of CpG-ODN per bird showed a significant increase in the lung APC population compared to the saline control group $(\sim 3$ and $\sim 4$ times higher, respectively) (Figures 1(a) and 1(b)). The highest APC percentage was seen with $50 \mu \mathrm{g}$ of CpG-ODN and lowest with the saline control. However, $5 \mu \mathrm{g}$ and $10 \mu \mathrm{g}$ of CpG-ODN treatment did not show a significant effect on APC population compared to the saline-treated control group in the lungs (Figures 1(a) and 1(b)). APCs (monocyte/macrophages) were further analyzed for the expression of maturation markers (CD40 costimulatory molecule) (Figure 1(c)). The CD40 signaling is well known to activate APCs and facilitate T-cell priming [43] to generate protective $\mathrm{CD}^{+}$cytotoxic T-cell (CTL) immunity. The mean fluorescence intensity (MFI) of CD40 expression on APCs, with each CpG-ODN dose tested, is shown in histograms (Figure 1(c)) and in the graph (Figure 1(d)). The MFI increased in a dose-dependent manner and was significantly high in both $25 \mu \mathrm{g}$ and $50 \mu \mathrm{g}$ groups (Figure $1(\mathrm{~d})$ ).
3.2.2. $\mathrm{CD}^{+}$and $\mathrm{CD} 8^{+}$T-Cell Subsets in the Lung. $\mathrm{CD} 4^{+}(\mathrm{T}$ helper cells) and $\mathrm{CD}^{+}$(cytotoxic T-cells) cells are the main types of T lymphocytes which play an important role in both humoral and cell-mediated immunity. We evaluated the effect of $\mathrm{CpG}-\mathrm{ODN}$ on $\mathrm{CD}^{+}$and $\mathrm{CD}^{+}$cell populations in the chicken lungs $72 \mathrm{hrs}$ post-CpG-ODN in ovo injections. Both $\mathrm{CD}^{+}$and $\mathrm{CD}^{+}$cell populations increased after CpGODN administration in a dose-dependent manner in the lungs (Figures $1(\mathrm{e})-1(\mathrm{~g})$ ). A significant increase in $\mathrm{CD}^{+}$ T-cells (Figure 1(f)), as well as a total number of T lymphocytes $\left(\mathrm{CD}^{+}\right.$and $\mathrm{CD}^{+}{ }^{+}$T-cells combined) (Figure $1(\mathrm{~g})$ ), was found in the lungs of chickens that received $25 \mu \mathrm{g}$ or $50 \mu \mathrm{g}$ CpG-ODN.

3.2.3. Histological Evaluation. Hematoxylin and eosin staining $72 \mathrm{hrs}$ post in ovo injections revealed higher cellular infiltration in the lungs of birds treated with $25 \mu \mathrm{g} \mathrm{CpG-}$ ODN (Figures 2(b), 2(d), and 2(f)) or $50 \mu \mathrm{g}$ CpG-ODN (data not shown), compared to the saline-treated group (Figures 2(a), 2(c), and 2(e)). The lungs of birds treated with CpG-ODN showed higher cellularity in the lung parenchyma (Figures 2(b), 2(d), and 2(f)), compared to saline-treated birds (Figures 2(a), 2(c), and 2(e)). Higher magnification of histology showed that these inflammatory cells were predominantly mononuclear cells with occasional heterophils (Figures 2(d) and 2(f)). There were no other inflammatory changes detected histologically suggesting no adverse effects or pathology is caused by any dose of CpG-ODN we used for this study.

\subsection{Immune Cell Profiling of the Spleen}

3.3.1. Antigen-Presenting Cells in the Spleen. Flow cytometry of splenocytes demonstrated the immune cell profile pattern similar to the lung samples. We observed a substantial effect of CpG-ODN treatment on MHCII-expressing APC cell population (monocyte/macrophages) in the spleen $72 \mathrm{hrs}$ post in ovo injections. The groups that received $25 \mu \mathrm{g}$ or $50 \mu \mathrm{g}$ of CpG-ODN per bird showed a significant increase in the splenic APC population compared to the saline control group $(\sim 3$ and $\sim 5$ times higher, respectively) (Figures 3(a) and 3(b)). In contrast, $5 \mu \mathrm{g}$ and $10 \mu \mathrm{g}$ of CpG-ODN treatment did not show a significant effect on APC population compared to the saline-treated control group in the lungs (Figures 3(a) and 3(b)). The MFI of CD40 expression on splenic APCs, with each CpG-ODN dose tested, are shown in histograms (Figure 3(c)) and in the graph (Figure 3(d)). The MFI increased in a dosedependent manner and was significantly high in both $25 \mu \mathrm{g}$ and $50 \mu \mathrm{g}$ groups (Figure $3(\mathrm{~d})$ ).

3.3.2. $C D 4^{+}$and $C D 8^{+}$T-Cell Subsets in the Spleen. We evaluated the effect of $\mathrm{CpG}-\mathrm{ODN}$ on $\mathrm{CD}^{+}$and $\mathrm{CD}^{+}$cell populations in the chicken spleen $72 \mathrm{hrs}$ post-CpG-ODN in ovo injections. We found a dose-dependent increase in both $\mathrm{CD}^{+}$and $\mathrm{CD}^{+}$cell populations in the spleen after $\mathrm{CpG}-$ ODN in ovo administration (Figures 3(e)-3(g)). A significant increase in $\mathrm{CD} 4^{+} \mathrm{T}$-cells (in $50 \mu \mathrm{g}$ group) and $\mathrm{CD} 8^{+} \mathrm{T}$-cells (both $25 \mu \mathrm{g}$ and $50 \mu \mathrm{g}$ groups) was observed (Figure 1(f)). However, the total number of $\mathrm{T}$ lymphocytes $\left(\mathrm{CD} 4^{+}\right.$and 


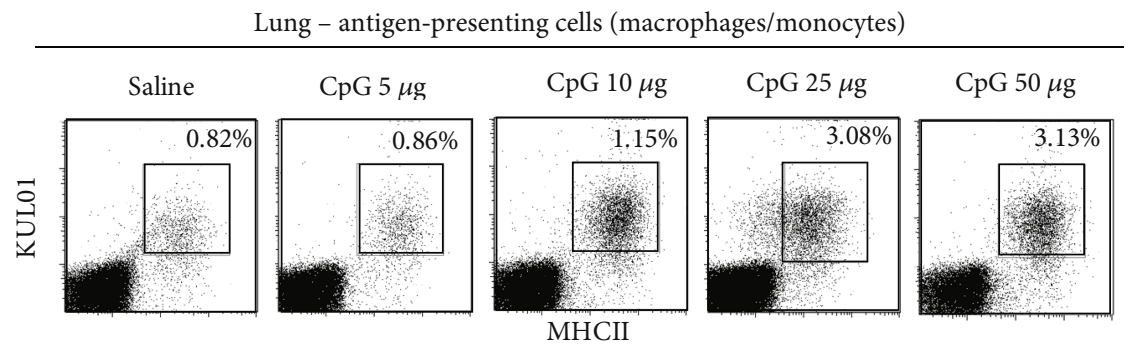

(a)

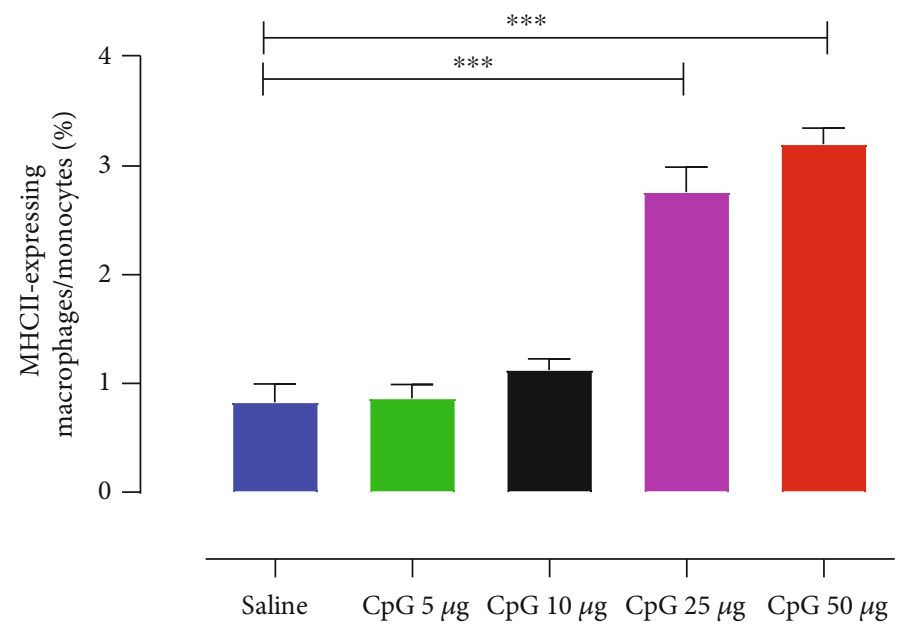

(b)

MHCII
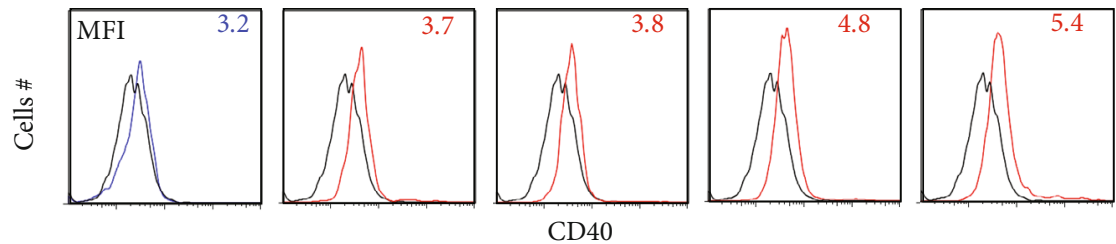

(c)

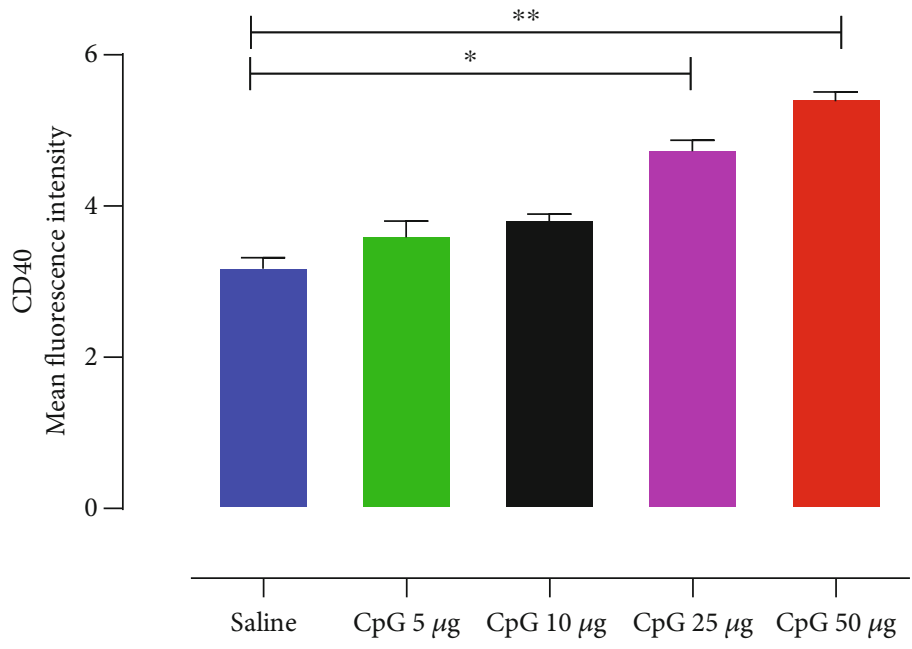

(d)

Figure 1: Continued. 
Lung $-\mathrm{CD} 4^{+}$and $\mathrm{CD} 8^{+}$T lymphocytes

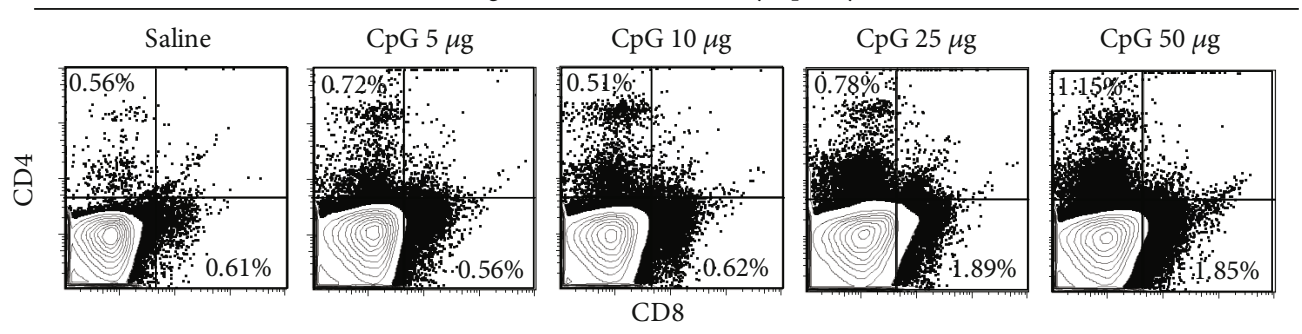

(e)

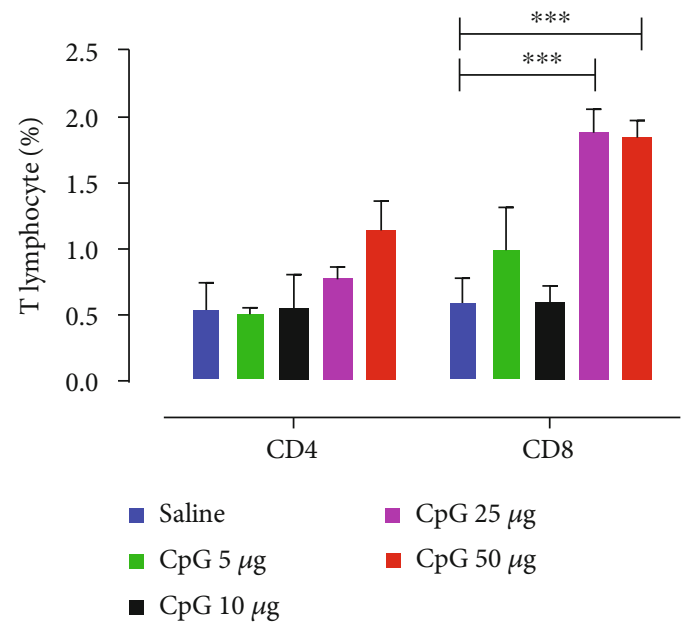

(f)

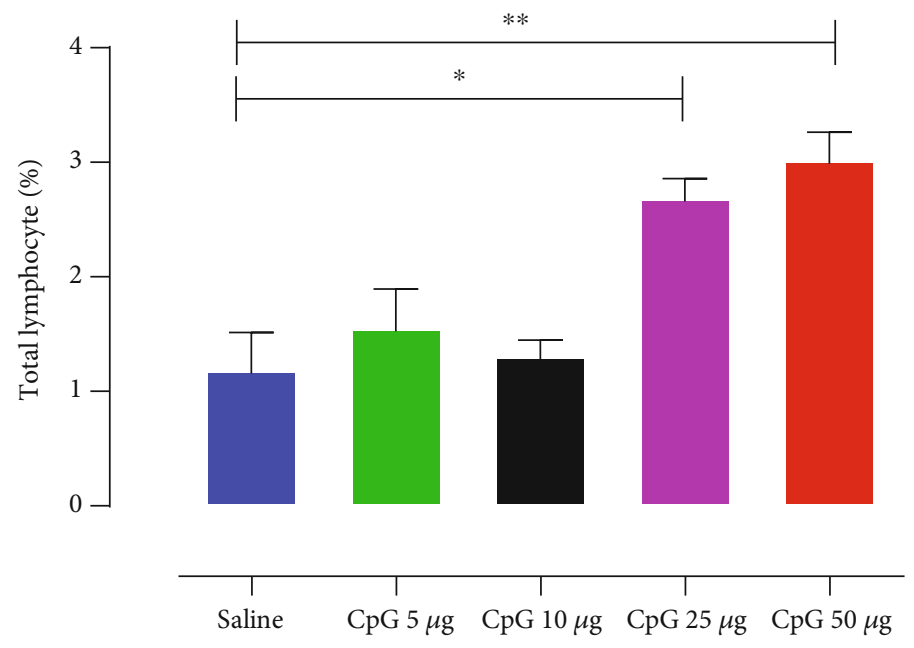

(g)

FIGURE 1: CpG-ODN-induced a dose-dependent enrichment of lung immunological niches. CpG-ODN treatment resulted in an increase in the number of infiltrating antigen-presenting cells and T lymphocytes in the lung. Flow cytometric analysis of lung cells at $72 \mathrm{hrs}$ post in ovo injections (day 21 embryo) ( $n=3-4 /$ group). First, cells were plotted based on forward vs. side scatter to gate monocyte/macrophage populations. Then, using PE-labeled mouse anti-chicken monocyte/macrophage (clone: KUL01) and AF488-labeled mouse MHC II monoclonal antibodies, the double-positive population was gated to quantify the number of APCs (dot plot panels, a). Bar diagrams (b) show the means of the total percentage of $\mathrm{MHC} \mathrm{II}^{+} \mathrm{APC}$ in various groups. Histogram panels (c) indicate the level of CD40 expression on MHC II ${ }^{+}$APCs (the MFI is indicated). Black histogram = isotype control, blue = saline, and red = CpG-ODN treated. Bar diagrams $(\mathrm{d})$ show the means of MFI of CD40 expression on MHC II ${ }^{+}$APC (vertical line and horizontal bar show the standard error of mean-SEM), $n=3-4$. Dunnett's test following ANOVA testing was used to test for significant differences between CpG-ODN doses and the saline control group. Asterisks indicate groups that were significantly different from the control group $(P<0.05)$. (MFI-geometric mean fluorescence intensity). (e-g) Flow cytometric analysis of lung T-cell populations in each group $(n=3-4)$ at $72 \mathrm{hr}$ post in ovo injections (day 1 hatch). $\mathrm{CD}^{+}$and $\mathrm{CD}^{+}$T-cells in the lung (dot plot panels, e) were quantified using PE-labeled mouse anti-chicken CD4 and FITC-labeled mouse anti-chicken CD8 monoclonal antibodies. Bar diagrams show $\mathrm{CD} 4^{+}$and $\mathrm{CD} 8^{+} \mathrm{T}$-cell number in the lungs (f), and the total number of $\mathrm{CD}^{+} \mathrm{T}$-cells and $\mathrm{CD} 8^{+} \mathrm{T}$-cells combined $(\mathrm{g})$ in various groups. Two-way ANOVA following the Bonferroni posttest was done when $\mathrm{CD}^{+}$and $\mathrm{CD} 8^{+}$cells were compared between $\mathrm{CpG}-\mathrm{ODN}$ received groups and saline control. Dunnett's test following ANOVA testing was used to test for significant differences in total T-cells between different CpG-ODN doses and the saline control group. Vertical line and horizontal bar show the standard error of mean-SEM. Asterisks indicate groups that were significantly different from the control group, ${ }^{*} P<0.05,{ }^{* *} P<0.01$, and ${ }^{* * *} P<0.001$.

$\mathrm{CD}^{+}$T-cells combined) (Figure $3(\mathrm{~g})$ ) was significantly high in the spleen of chickens that received $25 \mu \mathrm{g}$ or $50 \mu \mathrm{g} \mathrm{CpG-}$ ODN.

3.3.3. Histological Evaluation. Hematoxylin and eosin staining $72 \mathrm{hrs}$ post in ovo injections revealed higher cellular infiltration in the spleen of birds treated with $25 \mu \mathrm{g}$ CpG-ODN (Figures 4(b), 4(d), and 4(f)) or $50 \mu \mathrm{g}$ CpGODN (data not shown), compared to the saline-treated group (Figures 4(a), 4(c), and 4(e)). Higher magnification of histology showed that these inflammatory cells were predominantly mononuclear cells with occasional heterophils (Figures 4(d) and 4(f)). We did not observe any adverse effect or pathology in this study.

3.4. E. coli Challenge. The data of groups that received either $1 \times 10^{4}$ or $1 \times 10^{5} \mathrm{cfu}$ of $E$. coli were combined for clarity to present both the survival and the clinical score (CCS) 


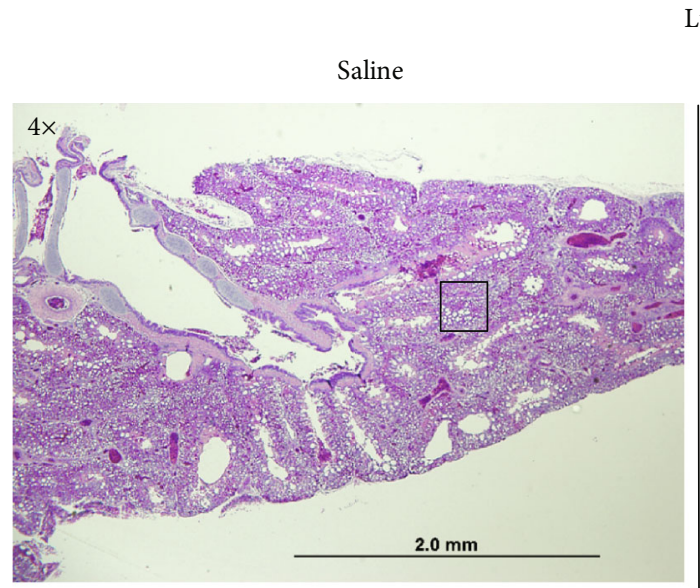

(a)

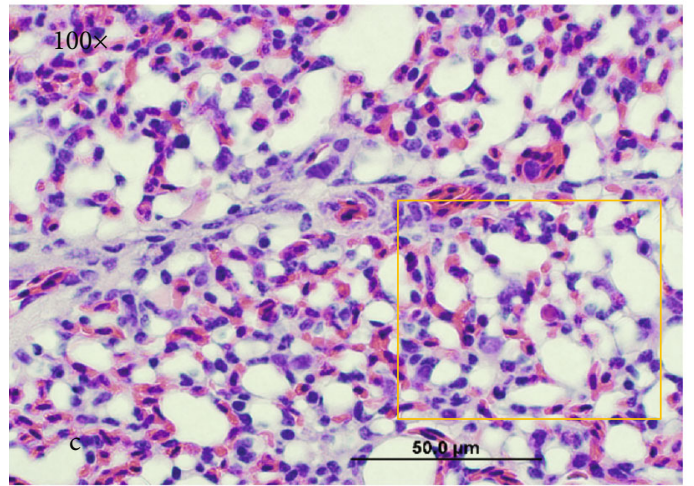

(c)

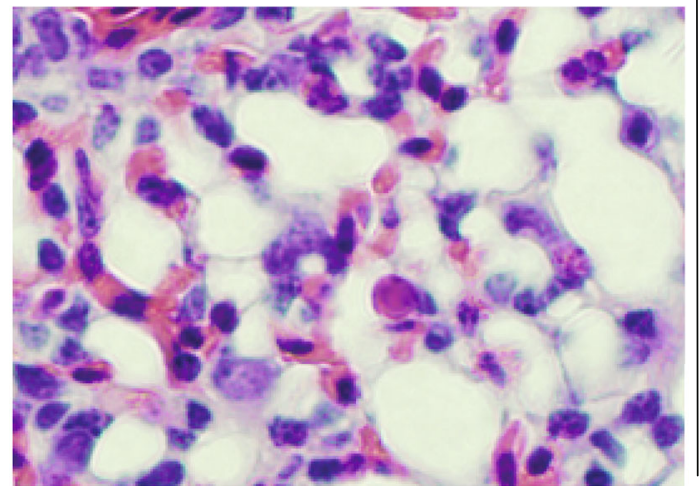

(e)
Lung

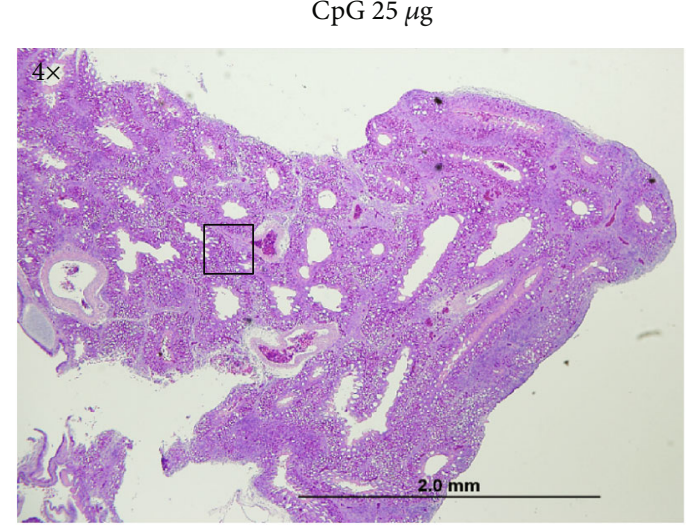

(b)

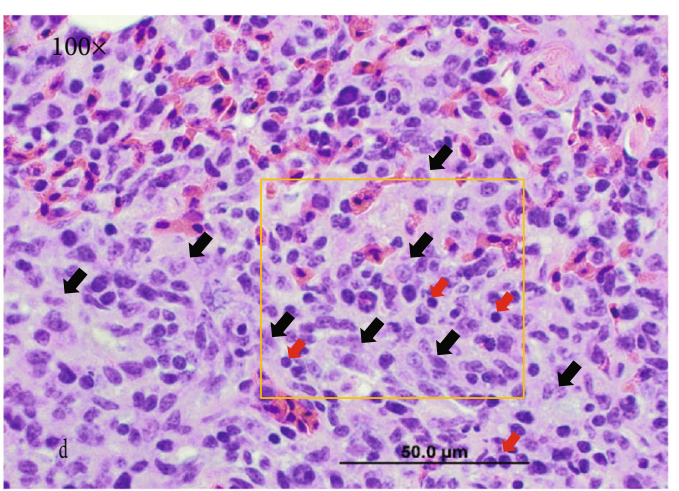

(d)

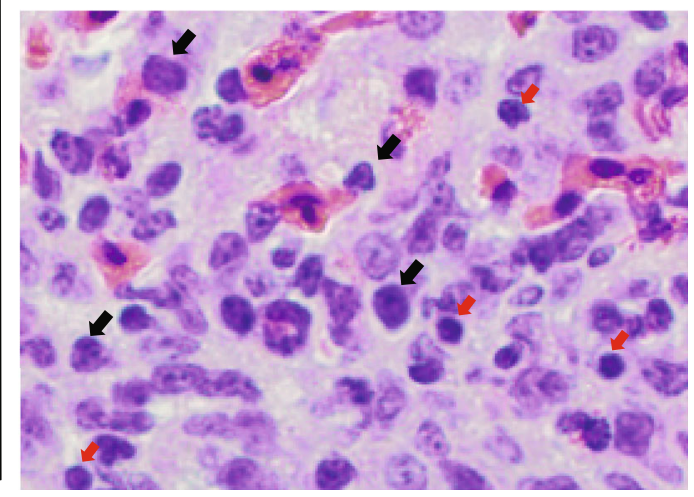

(f)

FIGURE 2: Histology of the lung. Hematoxylin and eosin staining 72 hrs post in ovo injections revealed higher cellular infiltration in the lungs of birds treated with $25 \mu \mathrm{g}$ CpG-ODN compared to saline control. Higher cellular infiltration was seen with CpG-ODN treatment (b) compared to saline (a) in low power (4x). High power magnification (100x) (d) and the magnified inset (f) showed that these cells mainly comprised of a mononuclear (macrophages) cell population (black arrows) and lymphocytes (red arrows).

analysis. In this study, survival following the E. coli challenge was significantly higher in groups of birds that received $25 \mu \mathrm{g}$ or $50 \mu \mathrm{g}$ of $\mathrm{CpG}-\mathrm{ODN}$ compared to the group of birds that received saline $(P=0.0323$ and 0.0430 , respectively $)$ (Figure 5(a)). Moreover, the reduction in the relative risk of mortality following the E. coli challenge was $48.5 \%$ and $47.5 \%$, respectively, in these group, indicating that increasing the dose beyond $25 \mu \mathrm{g}$ did not improve survival. This study revealed that the level of protection following the E. coli challenge is similar in birds that received 25 and $50 \mu \mathrm{g}$ of CpG-
ODN (Figure 5(b)). Birds that received $10 \mu \mathrm{g}$ of CpG-ODN tended to have higher survival than the saline control birds but were not statistically significant $(P=0.5957)$. The survival of birds that received $5 \mu \mathrm{g}$ of CpG-ODN was similar to the saline control group (Figure 5(a)).

The CCS for each bird was calculated by summing the daily scores throughout the 7-day observation period after the $E$. coli challenge. A significantly less CCS was recorded in $25 \mu \mathrm{g} \quad(P=0.0025)$ and $50 \mu \mathrm{g}(P=0.0016)$ of CpGODN-treated groups, compared to the saline-treated group 
Spleen-antigen-presenting cells (macrophages/monocytes)

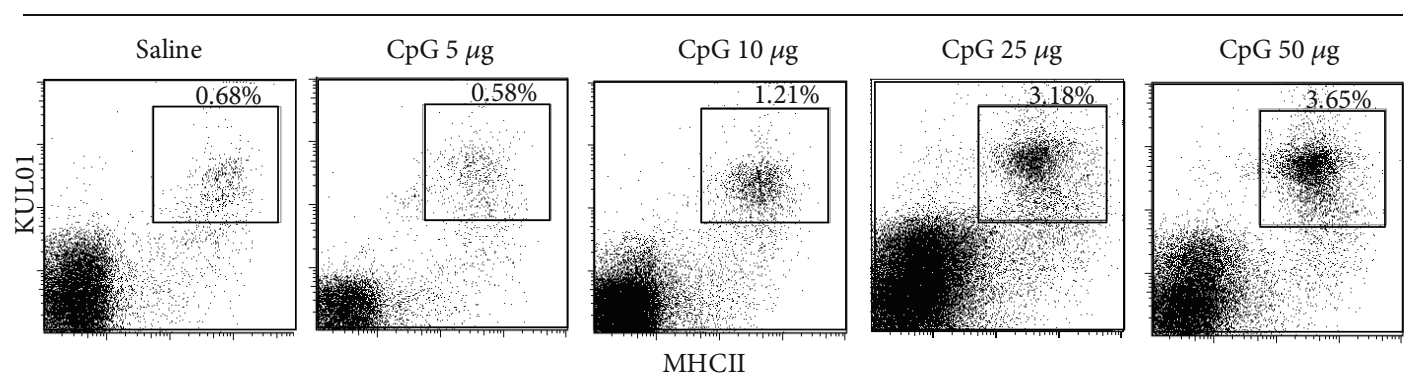

(a)

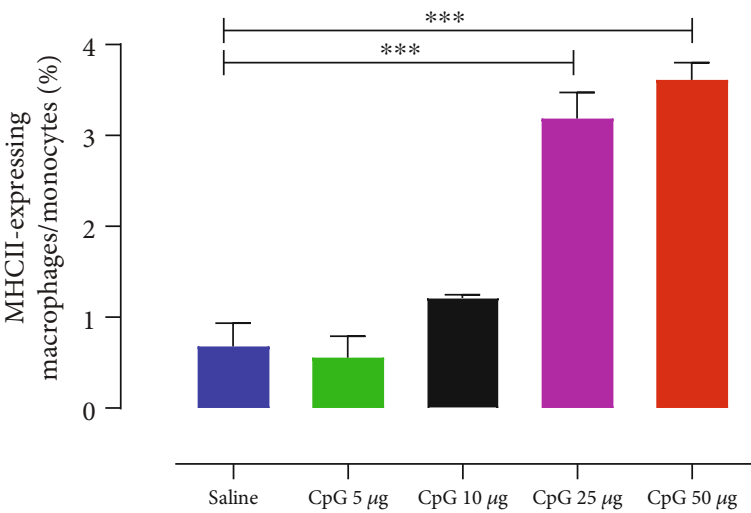

(b)
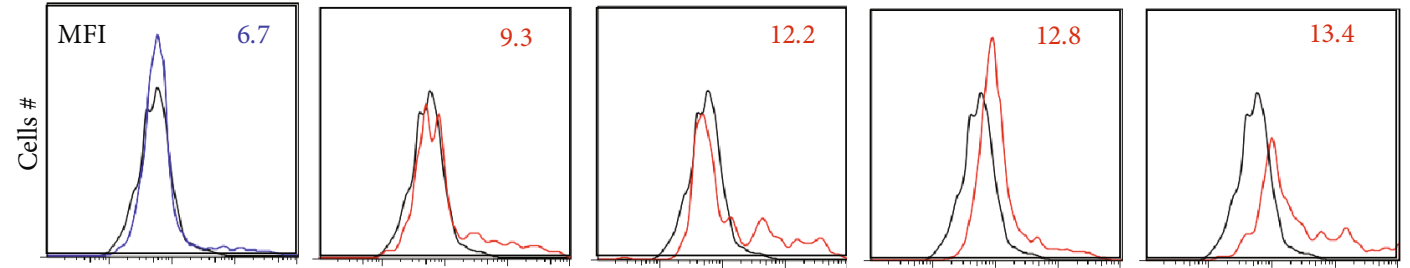

(c)

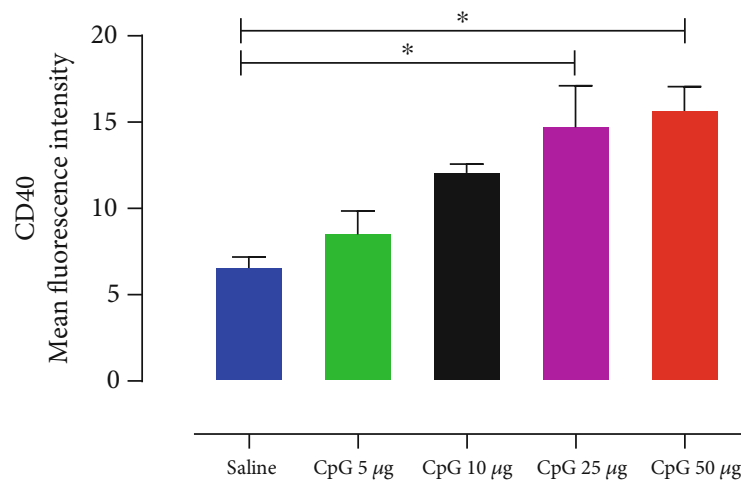

(d)

Figure 3: Continued. 
Spleen - CD4+ and CD8+ T lymphocytes

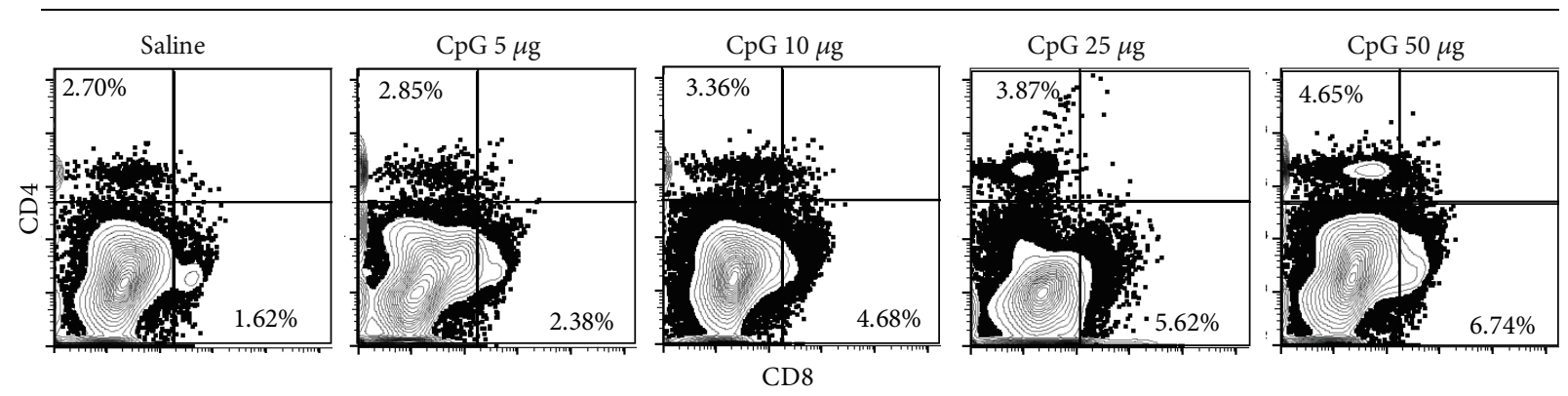

(e)

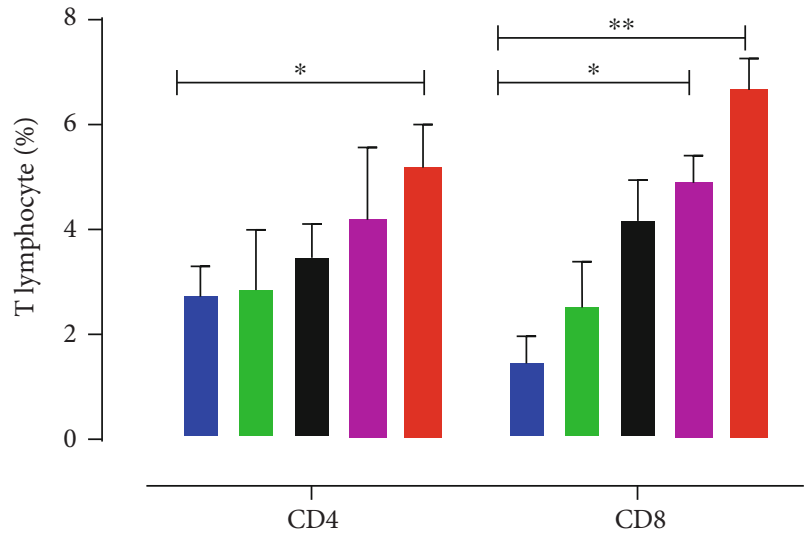

(f)

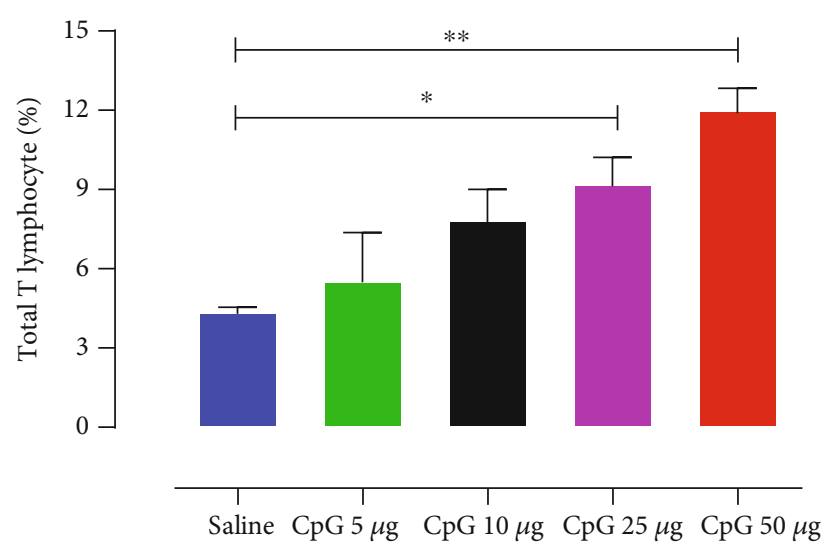

(g)

FIGURE 3: CpG-ODN induced a dose-dependent enrichment of splenic immunological niches. CpG-ODN treatment resulted in an increase in the number of infiltrating antigen-presenting cells and T lymphocytes in the spleen. Flow cytometric analysis of splenocytes at $72 \mathrm{hrs}$ post in ovo injections (day 21 embryo) ( $n=3$-4/group). Cells were plotted based on forward and side scatters to gate monocyte/macrophage populations. Then, using PE-labeled mouse anti-chicken monocyte/macrophage (clone: KUL01) and AF488-labeled mouse MHC II monoclonal antibodies, the double-positive population was gated to quantify the number of APCs (dot plot panels, a). Bar diagrams (b) show the means of the total percentage of $\mathrm{MHC}^{+} \mathrm{II}^{+} \mathrm{APC}$ in various groups. Histogram panels (c) indicate the level of CD40 expression on MHC II ${ }^{+}$APCs (the MFI is indicated). Black histogram = isotype control, blue = saline, and red = CpG-ODN treated. Bar diagrams (d) show the means of MFI of CD40 expression on MHC II ${ }^{+}$APC (vertical line and horizontal bar show the standard error of mean-SEM), $n=3-4$. Dunnett's test following ANOVA testing was used to test for significant differences between CpG-ODN doses and the saline control group. Asterisks indicate groups that were significantly different from the control group $(P<0.05)$. (MFI-geometric mean fluorescence intensity). (e-g) Flow cytometric analysis of the spleen T-cell populations in each group $(n=3-4)$ at $72 \mathrm{hr}$ post in ovo injections (day 1 hatch). $\mathrm{CD} 4^{+}$and $\mathrm{CD}^{+}$T-cells in the spleen (dot plot panels, e) were quantified using PE-labeled mouse anti-chicken CD4 and FITC-labeled mouse anti-chicken CD8 monoclonal antibodies. Bar diagrams show CD4 ${ }^{+}$and $\mathrm{CD}^{+} \mathrm{T}^{-}$-cell number in the spleen (f), and the total number of $\mathrm{CD}^{+} \mathrm{T}$-cells and $\mathrm{CD} 8^{+} \mathrm{T}$-cells combined $(\mathrm{g})$ in various groups. Two-way ANOVA following the Bonferroni posttest was done when $\mathrm{CD}^{+}$and $\mathrm{CD}^{+}$cells were compared between CpG-ODN received groups and saline control. Dunnett's test following ANOVA testing was used to test for significant differences in total T-cells between different CpG-ODN doses and the saline control group. Vertical line and horizontal bar show the standard error of mean-SEM. Asterisks indicate groups that were significantly different from the control group, ${ }^{*} P<0.05,{ }^{* *} P<0.01$, and ${ }^{* * *} P<0.001$.

(Figure 6(a)). The lowest CCSs were seen in birds that received $50 \mu \mathrm{g}$ of $\mathrm{CpG}-\mathrm{ODN}$ while the highest CCSs were detected from the saline control group (Figure 6(a)). Moreover, birds that received $\mathrm{CpG}-\mathrm{ODN}$ had lower amounts of bacteria isolated from air sac swabs compared to birds that received saline (Figure 6(b)). In comparison to other groups, birds that received $25 \mu \mathrm{g}$ or $50 \mu \mathrm{g}$ of CpG-ODN had significantly $(P<0.0001)$ less number of birds with $4+$ levels of bacterial growth. Birds that died or were euthanatized either had airsacculitis or pericarditis or a combination of airsacculitis together with pericarditis or polyserositis.

\section{Discussion}

While many preventive strategies are being implemented to minimize infectious diseases in poultry, they may not be sufficient to avert all pathogenic insults. Immune-based methods that stimulate innate immunity against a broad range of pathogens may provide a promising solution to disease problems in the poultry industry. CpG-ODN, a TLR agonist, is well known as an immune protective agent against bacterial $[28,29]$, viral $[44,45]$, and protozoal [25] infections in chickens. Furthermore, several studies have shown that CpG-ODN is effective as an immunostimulant in both 


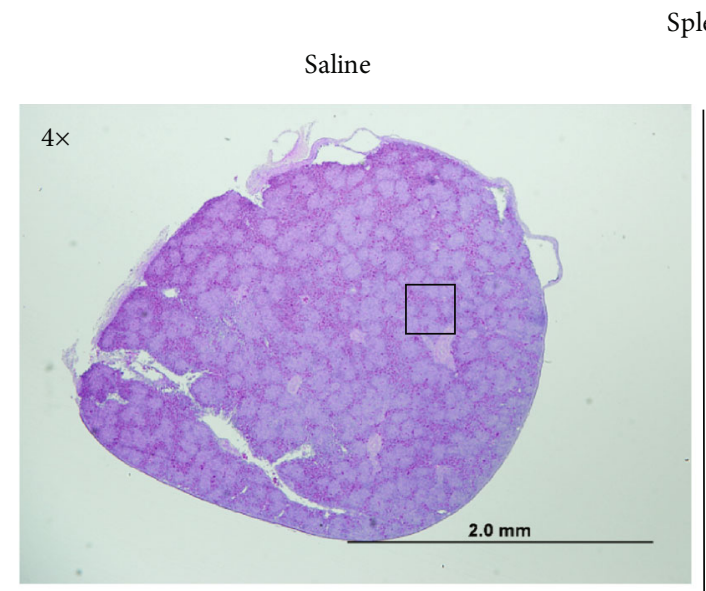

(a)

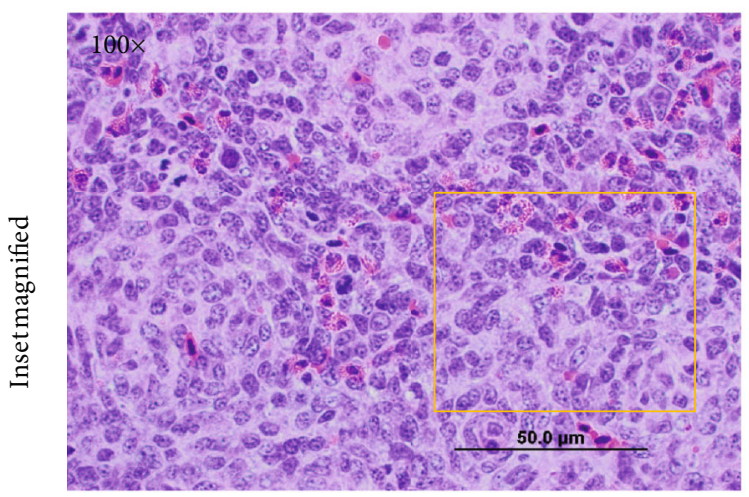

(c)

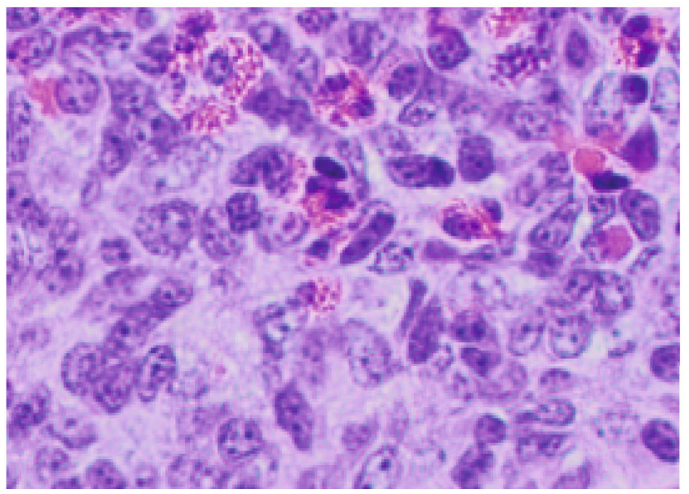

(e)

Spleen

CpG $25 \mu \mathrm{g}$

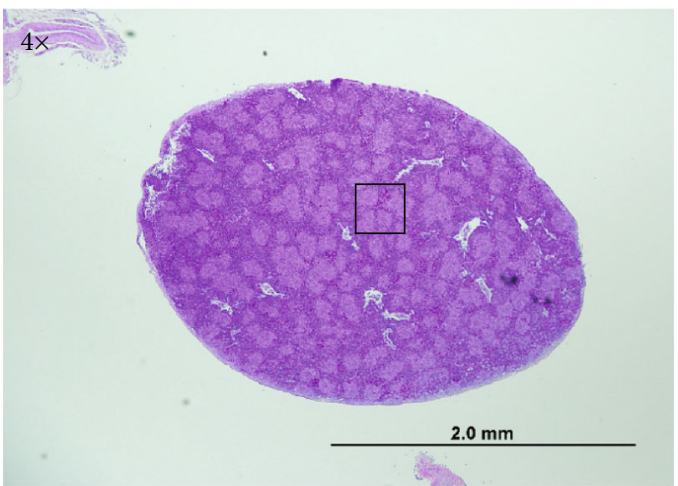

(b)

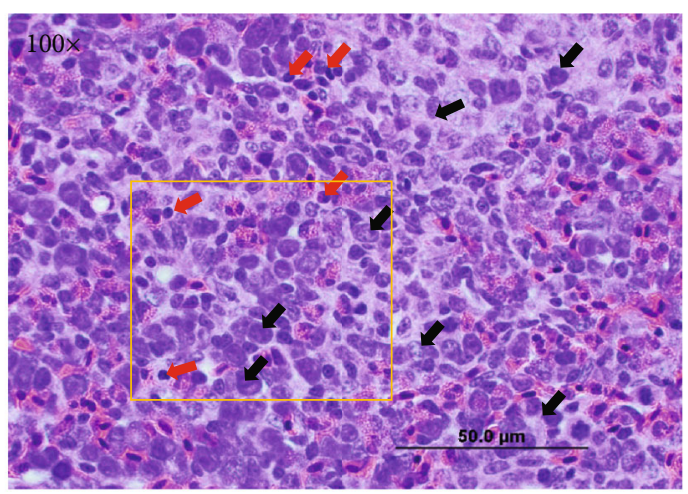

(d)

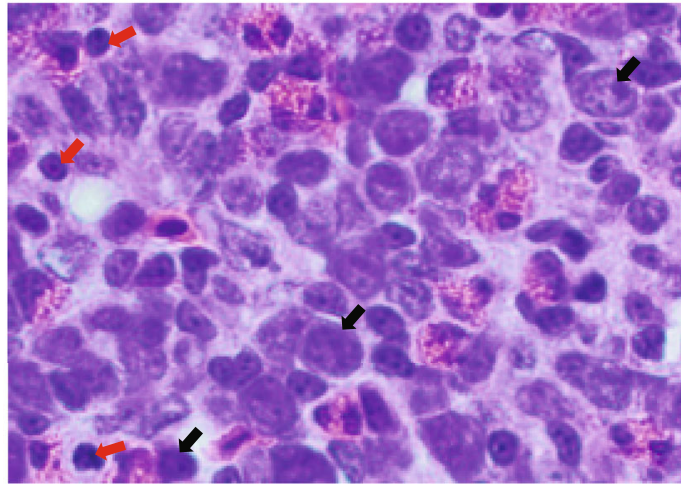

(f)

FIgURE 4: Histology of the spleen. Hematoxylin and eosin staining $72 \mathrm{hrs}$ post in ovo injections revealed higher cellular infiltration in the spleen of birds treated with $25 \mu \mathrm{g}$ CpG-ODN compared to saline control. Higher cellular infiltration was seen with CpG-ODN treatment (b) compared to saline (a) in low power (4x). High power magnification (100x) (d) and the magnified inset (f) showed that these cells mainly comprised of a mononuclear (macrophages) cell population (black arrows) and lymphocytes (red arrows).

mature and neonatal chickens against E. coli infections [4, $26,30]$. We have previously shown that CpG-ODN delivered through in ovo route, a most desirable and economical method of drug delivery in the chicken industry [46], can be effective in protecting neonatal chickens against bacterial diseases such as those caused by E. coli $[26,30]$ and Salmonella Typhimurium [29]. Other studies also reported the immunoprotective effect of CpG-ODN against Salmonella enteritidis [31, 32] and Campylobacter sp. [34]. The formulation of CpG-ODN with nanoparticles further improves the immune protective effect $[30,33,34]$. A recent study using boron nitride nanospheres functionalized with mesoporous silica demonstrated enhanced the delivery of $\mathrm{CpG}$ ODNs into macrophages that induced higher amounts of cytokines without cytotoxicity [47]. Previous studies suggested that enhanced expression of cytokines and chemokines [34, 35] and increased cellular functions, such as the increase in heterophil degranulation 


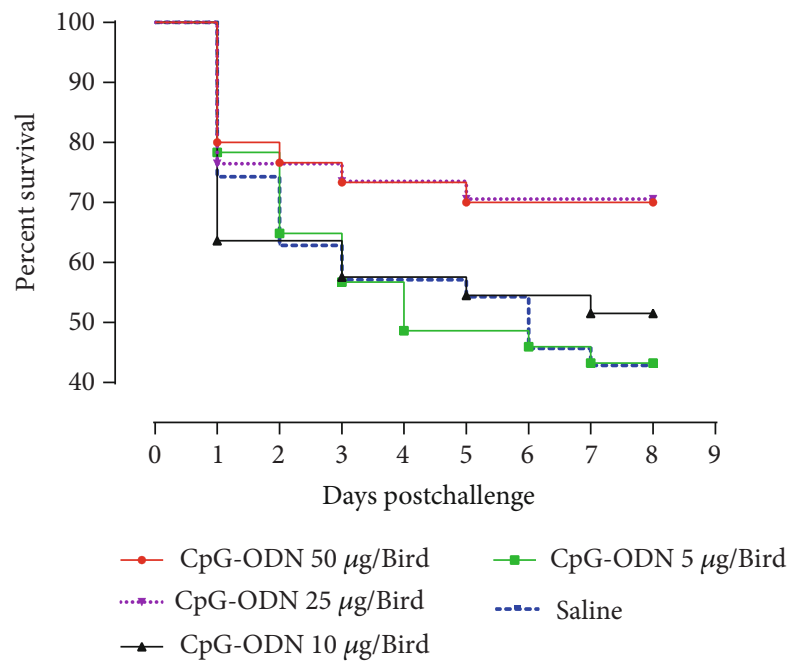

(a)

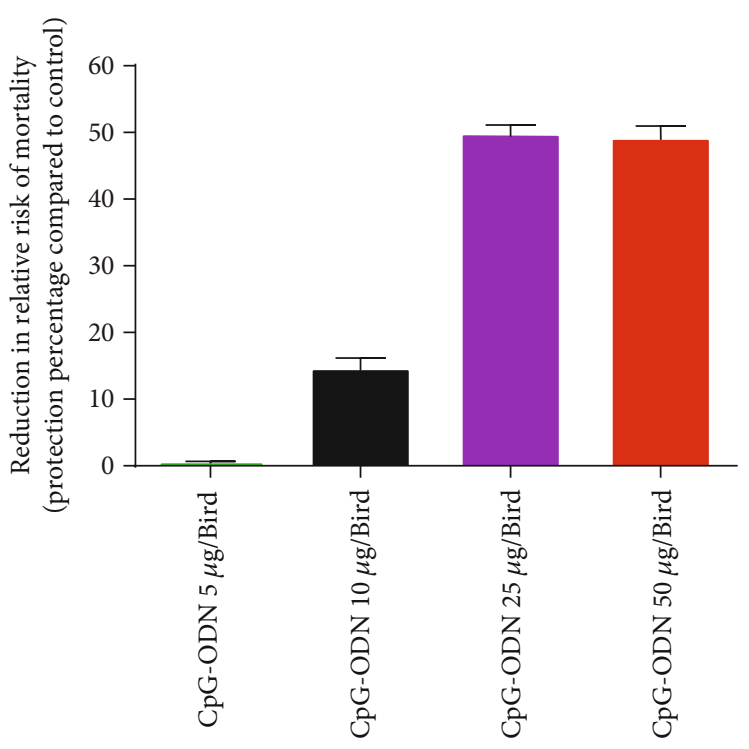

(b)

FIGURE 5: Survival pattern and reduction of the relative risk of mortality following the E.coli challenge. (a) Survival of broiler chickens following E. coli challenge. Groups of broiler chicken embryos at day 18 of incubation were injected with either 5, 10, 25, and 50 $\mu \mathrm{g}$ of CpG-ODN or sterile saline by the in ovo route and then challenged with either $1 \times 10^{5}$ or $1 \times 10^{4}$ cfu of E.coli three days later, at the day of hatch. Survival graph was plotted based on the event of a death in birds following challenge for each day postchallenge in each group. Survival was significantly higher in the birds that received $25 \mu \mathrm{g}$ or $50 \mu \mathrm{g}$ of CpG-ODN compared to the group of birds that received saline $(P=0.0323$ and 0.0430 , respectively). (b) The reduction of the relative risk of mortality compared to the saline control group following $E$. coli challenge.

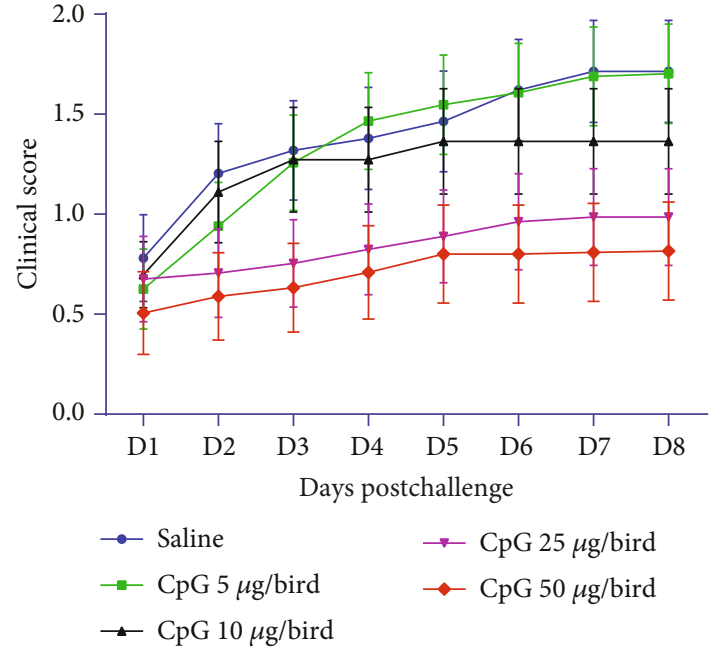

(a)

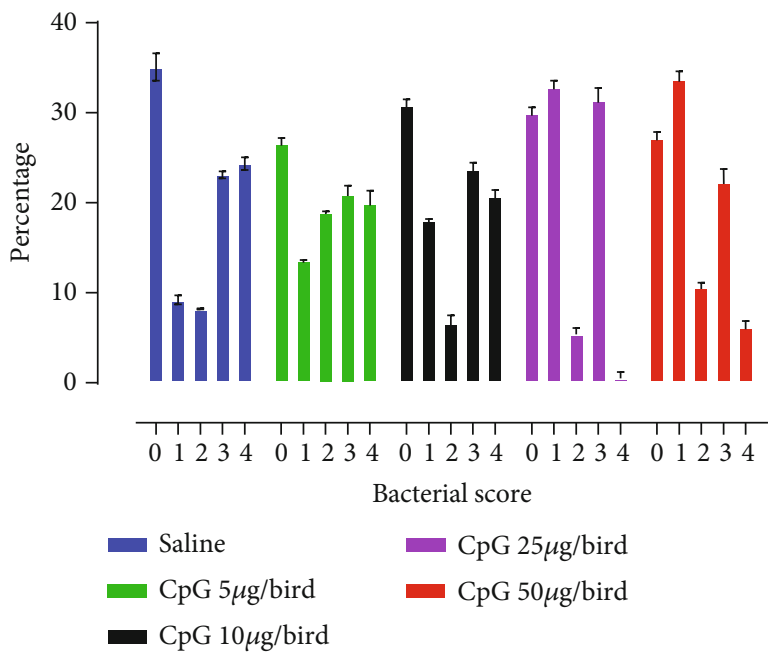

(b)

FIgURE 6: Clinical scores and bacterial growth following the E. coli challenge. (a) Clinical scores (CCS) of broiler chickens following E. coli challenge. Each data point represents the CCS of the individual group at that point (bar=mean with SEM). A significantly less CCS was recorded in $25 \mu \mathrm{g}(P=0.0025)$, and $50 \mu \mathrm{g}(P=0.0016)$ of CpG-ODN treated groups, compared to the saline-treated group. (b) Bar graph shows the percentage of birds in each treatment group that had each classification of bacterial growth. Bacterial swabs were taken from air sacs. Groups that received $25 \mu \mathrm{g}$ and $50 \mu \mathrm{g}$ of CpG-ODN showed significantly $(P<0.0001)$ less number of birds with higher levels $(4+)$ of bacterial growth compared to other groups.

and oxidative burst [48], play important roles in CpG-ODNinduced protection in chickens. We have recently reported an enhanced infiltration of inflammatory cells following an intrapulmonary delivery of CpG-ODN [4] and an enhanced enrichment of immunological niches after in ovo administra- tion of CpG-ODN [40] in chickens. Despite recent progress, the manner that $\mathrm{CpG}-\mathrm{ODN}$ alone confers immunoprotection against bacterial infection remains poorly understood.

The present study was undertaken to investigate CpGODN-mediated immune cell recruitment in the immunological 
niches in lymphoid (spleen) and nonlymphoid (lungs) organs in chickens and examine further if the immunological profiles correlate with protection against $E$. coli infection in chicks. In this study, we administered various doses of CpG-ODN through in ovo route (18-day-old embryonated eggs) and harvested the spleen and lungs at hatch to investigate CpG-ODN dose effect on the immunological profiles. One day after the hatch (four days after the CpG-ODN treatment), chicks were challenged with a virulent strain of E. coli to test protection from disease. Here, we examined the recruitment of macrophages/monocytes and $\mathrm{CD}^{+}$and $\mathrm{CD}^{+}$T-cell subsets in the immunological niches in the spleen and lungs. APCs, such as macrophages/monocytes, constitute essential components in the immune system, which process and present antigens, and initiate activation of effector immune cells. Maturation of APCs such as macrophages and dendritic cells involves an increased expression of costimulatory molecules such as CD80, CD86, and CD40 [49]. CD40 signaling on APC results in APC licensing (full activation) that facilitates $\mathrm{CD}^{+} \mathrm{T}$-cell priming [43] to orchestrate protective $\mathrm{CD}^{+}$cytotoxic T-cell (CTL) immunity [50]. Therefore, we examined the number of macrophages/monocytes present in the spleen and lungs as well as the expression of CD40 on these APCs in CpG-ODN treated or saline controls.

Our results showed a CpG-ODN-dose-dependent increase in the number of macrophages/monocytes in the immunological niches in both the lungs and the spleen. Moreover, we also observed an increase in CD40 expression with different doses of CpG-ODN. CD40 signaling is important for B cell proliferation, differentiation, T-cell proliferation, monocyte and dendritic cell growth, and cytokine production [51]. These data suggest that CpG-ODN administration not only enriches immune compartments with sentinel cells such as macrophages/monocytes that play important roles fighting against pathogens [52] but also activates them for proper maturation leading to the expression of a costimulatory molecule, CD40, which is well known for its role in the orchestration of immunity against pathogens [53]. These findings seen with CpG-ODN, such as immune cell activation and maturation, were comparable to the results of many earlier studies in other species [54-57]. The significant increase we observed in MHCII-expressing APCs with $25 \mu \mathrm{g}$ and $50 \mu \mathrm{g}$ of in ovo CpG-ODN in both the lungs and the spleen could suggest enhanced antigen presentation capability for an effective and rapid pathogen clearance in neonatal chicks. Also, we observed a dose-dependent increase in $\mathrm{CD}^{+}$and $\mathrm{CD}^{+} \mathrm{T}$-cell populations, which play important roles in humoral and cell-mediated immunity, in the spleen and lungs following CpG-ODN administration. These findings suggest that the immunological niches in CpG-ODN-treated chicks are well equipped with mature APCs with the ability to activate effector immune cells such as $\mathrm{CD}^{+}$and $\mathrm{CD}^{+} \mathrm{T}$ lymphocytes. The increase in the number of immune cells in the lungs and spleen can be attributed to either CpG-ODN-induced enhanced cytokinechemokine-mediated recruitment of immune cells or increased production of immune cells or both. We recently demonstrated that in ovo delivery of CpG-ODN enhanced the expression of proinflammatory cytokines, interleukin(IL-) $1 \beta$, IL-6, IL-18, and lipopolysaccharide-induced tumor necrosis factor (LITAF) in the lungs and spleen, suggesting that CpG-ODN promotes inflammatory responses in chickens. Accumulating evidence indicates that proinflammatory cytokines play a critical role in promoting strong antimicrobial immune responses.

Next, we investigated whether the levels of enrichment of immunological niches in the spleen and lungs by different doses of CpG-ODN have any correlation with the immunoprotection against $E$. coli infection. Therefore, we have assessed the clinical protection of neonatal chickens treated with various doses of CpG-ODN by challenging them with a virulent strain of $E$. coli. Here, we noted that the protection level of chicks that received $10 \mu \mathrm{g}$ or less CpG-ODN was not different from the chicks that received saline, consistent with our previous studies $[4,26,28,58]$. Chicks that received $25 \mu \mathrm{g}$ and $50 \mu \mathrm{g}$ of CpG-ODN were significantly protected compared to chicks that received saline. This protection correlates with the changes we detected in immune cell compartments in chickens at the time of hatch by different doses of CpG-ODN. We observed that $10 \mu \mathrm{g}$, but not $5 \mu \mathrm{g}$, CpG-ODN dose increased immune cells both in the spleen and in the lungs. However, both 5 and $10 \mu \mathrm{g}$ doses were not significantly different than the saline controls in immune profiles and protection. In contrast, chicks that received $25 \mu \mathrm{g}$ and $50 \mu \mathrm{g}$ of CpG-ODN demonstrated significantly enhanced MHCII-expressing APCs, CD40 expression on these APCs and T-cell populations that correlated with their ability to resist E. coli infection. Overall, our data established a cause and effect relationship between CpG-ODN-induced immune enrichment and the antibacterial immunity and suggest that CpG-ODNs not only stimulate immune cells but also remarkably enriches the immunological niche, making neonatal chicks well prepared to tackle pathogenic insults.

\section{Abbreviations}

CpG-ODN: Oligodeoxynucleotides containing CpG motifs

PAMPs: $\quad$ Pathogen-associated molecular patterns

PRR: Pattern recognition receptors

TLRs: $\quad$ Toll-like receptors

DC: $\quad$ Dendritic cells

DMEM: Dulbecco's modified Eagle medium

APCs: Antigen-presenting cells

CCSs: $\quad$ Cumulative clinical scores

CTL: $\quad \mathrm{CD}^{+}$cytotoxic T-cell.

\section{Data Availability}

The data used to support the findings of this study are available from the corresponding author upon request.

\section{Conflicts of Interest}

The authors declare that they have no conflicts of interest. 


\section{Acknowledgments}

We wish to thank the Animal Care Unit staff at the Western College of Veterinary Medicine, University of Saskatchewan. Financial support was provided by grants from the Natural Sciences and Engineering Research Council of Canada (417227), the Western Economic Diversification Canada (416876), the Saskatchewan Chicken Industry Development Fund (417229), the Canadian Poultry Research Council (417158), and the Alberta Livestock and Meat Agency Ltd (416490).

\section{References}

[1] J. J. Dibner, C. D. Knight, M. L. Kitchell, C. A. Atwell, A. C. Downs, and F. J. Ivey, "Early Feeding and Development of the Immune System in Neonatal Poultry ${ }^{1}$," Journal of Applied Poultry Research, vol. 7, no. 4, pp. 425-436, 1998.

[2] L. K. N. H. John Barnes and J.-P. Vaillancourt, Diseases of Poultry, A. M. F. Y. M. Saif, J. R. Glisson, L. R. McDougald, L. K. Nolan, and D. E. Swayne, Eds., Blacwell Publishing Professional, Iowa, 2008, chap. 18 Colibacillosis.

[3] H. Yassin, A. G. J. Velthuis, M. Boerjan, and J. van Riel, "Field study on broilers' first-week mortality," Poultry Science, vol. 88, no. 4, pp. 798-804, 2009.

[4] K. B. Goonewardene, S. Popowich, T. Gunawardana et al., "Intrapulmonary delivery of CpG-ODN microdroplets provides protection against Escherichia coli septicemia in neonatal broiler chickens," Avian Diseases, vol. 61, no. 4, pp. 503511, 2017.

[5] M. Casewell, C. Friis, E. Marco, P. McMullin, and I. Phillips, "The European ban on growth-promoting antibiotics and emerging consequences for human and animal health," Journal of Antimicrobial Chemotherapy, vol. 52, no. 2, pp. 159161, 2003.

[6] C. L. Gyles, "Antimicrobial resistance in selected bacteria from poultry," Animal Health Research Reviews, vol. 9, no. 2, pp. 149-158, 2008.

[7] J. P. Graham, J. J. Boland, and E. Silbergeld, "Growth promoting antibiotics in food animal production: an economic analysis," Public Health Reports, vol. 122, no. 1, pp. 79-87, 2007.

[8] A. Agunos, R. K. Arsenault, B. P. Avery et al., "Changes in antimicrobial resistance levels among Escherichia coli, Salmonella, and Campylobacter in Ontario broiler chickens between 2003 and 2015," Canadian Journal of Veterinary Research, vol. 82, no. 3, pp. 163-177, 2018.

[9] S. Millet and L. Maertens, "The European ban on antibiotic growth promoters in animal feed: from challenges to opportunities," The Veterinary Journal, vol. 187, no. 2, pp. 143-144, 2011.

[10] H. K. Allen, U. Y. Levine, T. Looft, M. Bandrick, and T. A. Casey, "Treatment, promotion, commotion: antibiotic alternatives in food-producing animals," Trends in Microbiology, vol. 21, no. 3, pp. 114-119, 2013.

[11] A. M. Krieg, "CPG motifs INBACTERIALDNAANDTHEIRIMMUNEEFFECTS," Annual Review of Immunology, vol. 20, no. 1, pp. 709760, 2002.

[12] A. Aderem and R. J. Ulevitch, "Toll-like receptors in the induction of the innate immune response," Nature, vol. 406, no. 6797 , pp. $782-787,2000$.
[13] A. Makkouk and A. M. Abdelnoor, "The potential use of tolllike receptor (TLR) agonists and antagonists as prophylactic and/or therapeutic agents," Immunopharmacology and Immunotoxicology, vol. 31, no. 3, pp. 331-338, 2009.

[14] A. M. Krieg, "Toll-like receptor 9 (TLR9) agonists in the treatment of cancer," Oncogene, vol. 27, no. 2, pp. 161167, 2008.

[15] D. W. Yeh, Y. L. Liu, Y. C. Lo et al., “Toll-like receptor 9 and 21 have different ligand recognition profiles and cooperatively mediate activity of CpG-oligodeoxynucleotides in zebrafish," Proceedings of the National Academy of Sciences, vol. 110, no. 51, pp. 20711-20716, 2013.

[16] G. Hartmann, R. D. Weeratna, Z. K. Ballas et al., "Delineation of a CpG phosphorothioate oligodeoxynucleotide for activating primate immune responses in vitro and in vivo," Journal of immunology, vol. 164, no. 3, pp. 1617-1624, 2000.

[17] H. Zhang, W. Chen, K. Gong, and J. Chen, "Nanoscale zeolitic imidazolate framework- 8 as efficient vehicles for enhanced delivery of CpG oligodeoxynucleotides," ACS Applied Materials \& Interfaces, vol. 9, no. 37, pp. 31519-31525, 2017.

[18] H. Zhang and X. D. Gao, "Nanodelivery systems for enhancing the immunostimulatory effect of CpG oligodeoxynucleotides," Materials Science and Engineering: C, vol. 70, Part 2, pp. 935946, 2017.

[19] H. C. Cho, B. H. Kim, K. Kim, J. Y. Park, J. H. Chang, and S. K. Kim, "Cancer immunotherapeutic effects of novel CpG ODN in murine tumor model," Int Immunopharmacol, vol. 8, no. 10, pp. 1401-1407, 2008.

[20] J. B. Jørgensen, L.-H. Johansen, K. Steiro, and A. Johansen, "CpG DNA induces protective antiviral immune responses in Atlantic salmon (Salmo salar L.)," Journal of Virology, vol. 77, no. 21, pp. 11471-11479, 2003.

[21] A. K. Nichani, A. Mena, Y. Popowych et al., "In vivo immunostimulatory effects of CpG oligodeoxynucleotide in cattle and sheep," Veterinary Immunology and Immunopathology, vol. 98, no. 1-2, pp. 17-29, 2004.

[22] J. Adamsson, M. Lindblad, A. Lundqvist, D. Kelly, J. Holmgren, and A. M. Harandi, "Novel immunostimulatory agent based on CpG oligodeoxynucleotide linked to the nontoxic B subunit of cholera toxin," The Journal of Immunology, vol. 176, no. 8, pp. 4902-4913, 2006.

[23] G. J. Weiner, H. M. Liu, J. E. Wooldridge, C. E. Dahle, and A. M. Krieg, "Immunostimulatory oligodeoxynucleotides containing the $\mathrm{CpG}$ motif are effective as immune adjuvants in tumor antigen immunization," Proceedings of the National Academy of Sciences U S A, vol. 94, no. 20, pp. 10833-10837, 1997.

[24] W. Meng, T. Yamazaki, Y. Nishida, and N. Hanagata, "Nuclease-resistant immunostimulatory phosphodiester CpG oligodeoxynucleotides as human Toll-like receptor 9 agonists," BMC Biotechnology, vol. 11, no. 1, p. 88, 2011.

[25] R. A. Dalloul, H. S. Lillehoj, M. Okamura et al., "In vivo effects of $\mathrm{CpG}$ oligodeoxynucleotide on Eimeria infection in chickens," Avian Diseases, vol. 48, no. 4, pp. 783-790, 2004.

[26] S. Gomis, L. Babiuk, B. Allan et al., "Protection of neonatal chicks against a lethal challenge of Escherichia coli using DNA containing cytosine-phosphodiester-guanine motifs," Avian Diseases, vol. 48, no. 4, pp. 813-822, 2004.

[27] T. Maurer, C. Pournaras, J. A. Aguilar-Pimentel et al., "Immunostimulatory CpG-DNA and PSA-peptide vaccination elicits profound cytotoxic T cell responses," Urologic Oncology: 
Seminars and Original Investigations, vol. 31, no. 7, pp. 13951401, 2013.

[28] S. Gomis, L. Babiuk, D. L. Godson et al., "Protection of chickens against Escherichia coli infections by DNA containing CpG motifs," Infection and Immunity, vol. 71, no. 2, pp. 857-863, 2003.

[29] A. Taghavi, B. Allan, G. Mutwiri et al., "Protection of neonatal broiler chicks against Salmonella Typhimurium septicemia by DNA containing CpG motifs," Avian Diseases, vol. 52, no. 3, pp. 398-406, 2008.

[30] T. Gunawardana, M. Foldvari, T. Zachar et al., "Protection of Neonatal Broiler Chickens Following in ovo Delivery of Oligodeoxynucleotides Containing CpG Motifs (CpG-ODN) Formulated with Carbon Nanotubes or Liposomes," Avian Diseases, vol. 59, no. 1, pp. 31-37, 2015.

[31] H. He, V. K. Lowry, C. L. Swaggerty, P. J. Ferro, and M. H. Kogut, "In vitro activation of chicken leukocytes and in vivo protection against Salmonella enteritidis organ invasion and peritoneal S. enteritidis infection-induced mortality in neonatal chickens by immunostimulatory CpG oligodeoxynucleotide," FEMS Immunology \& Medical Microbiology, vol. 43, no. 1, pp. 81-89, 2005.

[32] K. M. Mackinnon, H. He, C. L. Swaggerty et al., "In ovo treatment with $\mathrm{CpG}$ oligodeoxynucleotides decreases colonization of Salmonella enteriditis in broiler chickens," Veterinary Immunology and Immunopathology, vol. 127, no. 3-4, pp. 371-375, 2009.

[33] A. Taghavi, B. Allan, G. Mutwiri et al., "Enhancement of immunoprotective effect of CpG-ODN by formulation with polyphosphazenes against E. coli septicemia in neonatal chickens," Current Drug Delivery, vol. 6, no. 1, pp. 76-82, 2009.

[34] K. Taha-Abdelaziz, T. N. Alkie, D. C. Hodgins, A. Yitbarek, B. Shojadoost, and S. Sharif, "Gene expression profiling of chicken cecal tonsils and ileum following oral exposure to soluble and PLGA-encapsulated CpG ODN, and lysate of Campylobacter jejuni," Veterinary Microbiology, vol. 212, pp. 6774, 2017.

[35] A. Dar, S. Tikoo, A. Potter et al., "CpG-ODNs induced changes in cytokine/chemokines genes expression associated with suppression of infectious bronchitis virus replication in chicken lungs," Veterinary Immunology and Immunopathology, vol. 160, no. 3-4, pp. 209-217, 2014.

[36] R. K. Beal, C. Powers, T. F. Davison, P. A. Barrow, and A. L. Smith, "Clearance of enteric Salmonella enterica serovar Typhimurium in chickens is independent of B-cell function," Infection and Immunity, vol. 74, no. 2, pp. 1442-1444, 2006.

[37] A. Andoh, H. Takaya, T. Saotome et al., "Cytokine regulation of chemokine (IL-8, MCP-1, and RANTES) gene expression in human pancreatic periacinar myofibroblasts," Gastroenterology, vol. 119, no. 1, pp. 211-219, 2000.

[38] J. R. Bradley, "TNF-mediated inflammatory disease," The Journal of Pathology, vol. 214, no. 2, pp. 149-160, 2008.

[39] X. Wang, G. Z. Feuerstein, J. L. Gu, P. G. Lysko, and T. L. Yue, "Interleukin- $1 \beta$ induces expression of adhesion molecules in human vascular smooth muscle cells and enhances adhesion of leukocytes to smooth muscle cells," Atherosclerosis, vol. 115, no. 1, pp. 89-98, 1995.

[40] T. Gunawardana, K. A. Ahmed, K. Goonewardene et al., "Synthetic CpG-ODN rapidly enriches immune compartments in neonatal chicks to induce protective immunity against bacterial infections," Scientific Reports, vol. 9, no. 1, p. 341, 2019.

[41] K. A. Ahmed, L. Wang, P. Griebel, D. D. Mousseau, and J. Xiang, "Differential expression of mannose-6-phosphate receptor regulates T cell contraction," Journal of Leukocyte Biology, vol. 98, no. 3, pp. 313-318, 2015.

[42] K. A. Ahmed and J. Xiang, "mTORC1 regulates mannose-6phosphate receptor transport and T-cell vulnerability to regulatory T cells by controlling kinesin KIF13A," Cell Discovery, vol. 3, no. 1, 2017.

[43] S. P. Schoenberger, R. E. M. Toes, E. I. H. van der Voort, R. Offringa, and C. J. M. Melief, "T-cell help for cytotoxic T lymphocytes is mediated by CD40-CD40L interactions," Nature, vol. 393, no. 6684, pp. 480-483, 1998.

[44] A. Dar, A. Potter, S. Tikoo et al., "CpG oligodeoxynucleotides activate innate immune response that suppresses infectious bronchitis virus replication in chicken embryos," Avian Diseases, vol. 53, no. 2, pp. 261-267, 2009.

[45] S. Thapa, M. Cader, K. Murugananthan et al., "In ovo delivery of CpG DNA reduces avian infectious laryngotracheitis virus induced mortality and morbidity," Viruses, vol. 7, no. 4, pp. 1832-1852, 2015.

[46] C. A. Ricks, A. Avakian, T. Bryan et al., Advances in Veterinary Medicine, D. S. Ronald, Ed., vol. 41, Academic Press, 1999.

[47] H. Zhang, T. Cheng, L. Lai et al., "BN nanospheres functionalized with mesoporous silica for enhancing $\mathrm{CpG}$ oligodeoxynucleotide-mediated cancer immunotherapy," Nanoscale, vol. 10, no. 30, pp. 14516-14524, 2018.

[48] H. He, K. J. Genovese, C. L. Swaggerty, D. J. Nisbet, and M. H. Kogut, "In vivo priming heterophil innate immune functions and increasing resistance to Salmonella enteritidis infection in neonatal chickens by immune stimulatory CpG oligodeoxynucleotides," Veterinary Immunology and Immunopathology, vol. 117, no. 3-4, pp. 275-283, 2007.

[49] K. Hoebe, E. Janssen, and B. Beutler, "The interface between innate and adaptive immunity," Nature Immunology, vol. 5, no. 10, pp. 971-974, 2004.

[50] M. F. Mackey, J. R. Gunn, C. Maliszewsky, H. Kikutani, R. J. Noelle, and R. J. Barth Jr., "dendritic cells require maturation via CD40 to generate protective antitumor immunity," The Journal of Immunology, vol. 161, no. 5, pp. 2094-2098, 1998.

[51] C. V. Kooten and J. Banchereau, "Functions of CD40 on B cells, dendritic cells and other cells," Current Opinion in Immunology, vol. 9, no. 3, pp. 330-337, 1997.

[52] M. Benoit, B. Desnues, and J. L. Mege, "Macrophage polarization in bacterial infections," The Journal of Immunology, vol. 181, no. 6, pp. 3733-3739, 2008.

[53] K. A. Ahmed, L. Wang, M. A. Munegowda et al., "Direct in vivo evidence of $\mathrm{CD} 4+\mathrm{T}$ cell requirement for CTL response and memory via pMHC-I targeting and CD40L signaling," Journal of Leukocyte Biology, vol. 92, no. 2, pp. 289-300, 2012.

[54] T. Sparwasser, E.-S. Koch, R. M. Vabulas et al., "Bacterial DNA and immunostimulatory $\mathrm{CpG}$ oligonucleotides trigger maturation and activation of murine dendritic cells," European Journal of Immunology, vol. 28, no. 6, pp. 2045-2054, 1998.

[55] H. Häcker, R. M. Vabulas, O. Takeuchi, K. Hoshino, S. Akira, and H. Wagner, "Immune cell activation by bacterial CpgDNA through myeloid differentiation marker 88 and tumor necrosis factor receptor-associated factor (Traf)6," The Journal of Experimental Medicine, vol. 192, no. 4, pp. 595-600, 2000 . 
[56] T. L. Roberts, J. A. Dunn, T. D. Terry et al., "Differences in macrophage activation by bacterial DNA and CpGcontaining oligonucleotides," The Journal of Immunology, vol. 175, no. 6, pp. 3569-3576, 2005.

[57] Z. Meng, J. Shao, and L. Xiang, "CpG oligodeoxynucleotides activate grass carp (Ctenopharyngodon idellus) macrophages," Developmental \& Comparative Immunology, vol. 27, no. 4, pp. 313-321, 2003.

[58] S. Gomis, L. Babiuk, B. Allan et al., "Protection of chickens against a lethal challenge of Escherichia coli by a vaccine containing CpG oligodeoxynucleotides as an adjuvant," Avian Diseases, vol. 51, no. 1, pp. 78-83, 2007. 


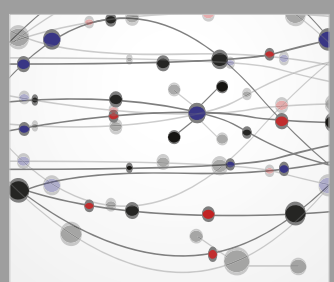

The Scientific World Journal
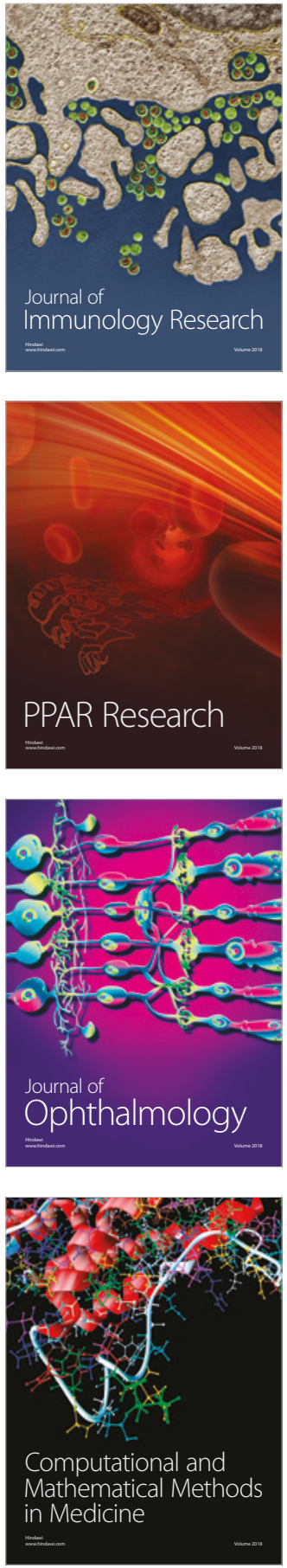

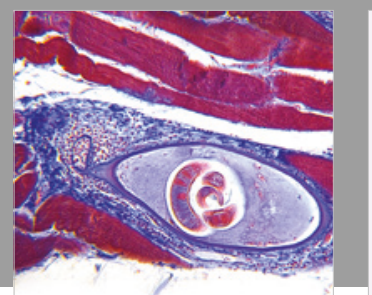

Gastroenterology Research and Practice

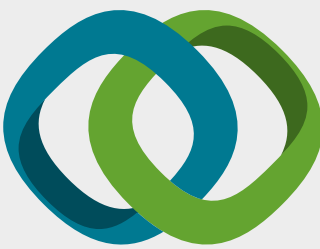

\section{Hindawi}

Submit your manuscripts at

www.hindawi.com
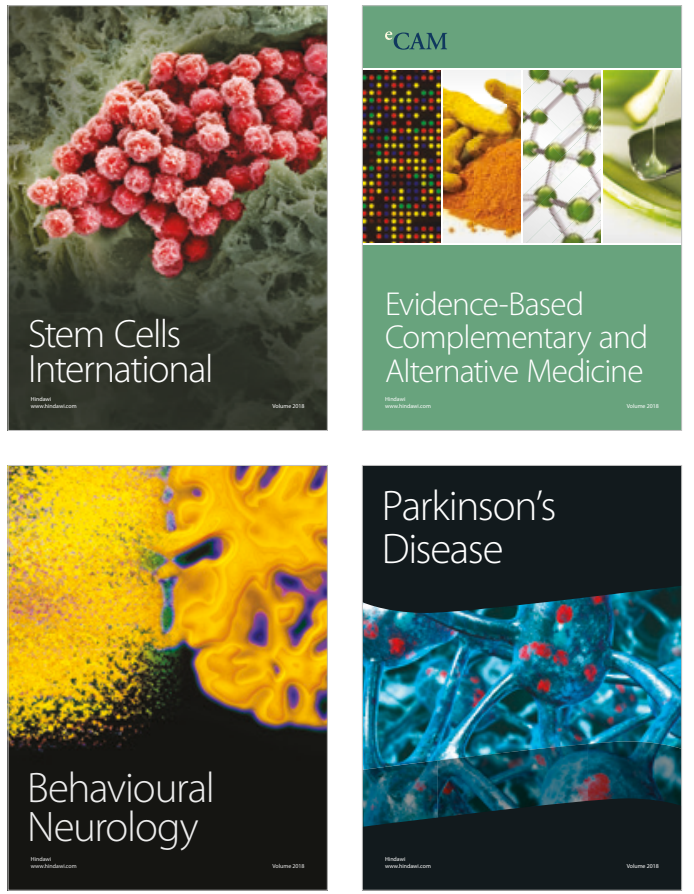

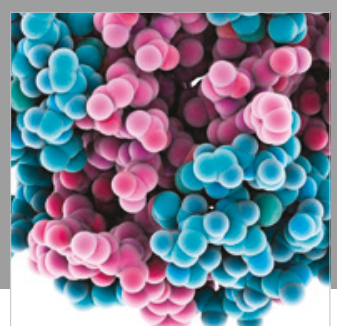

ournal of

Diabetes Research

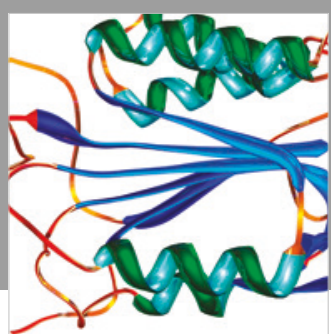

Disease Markers
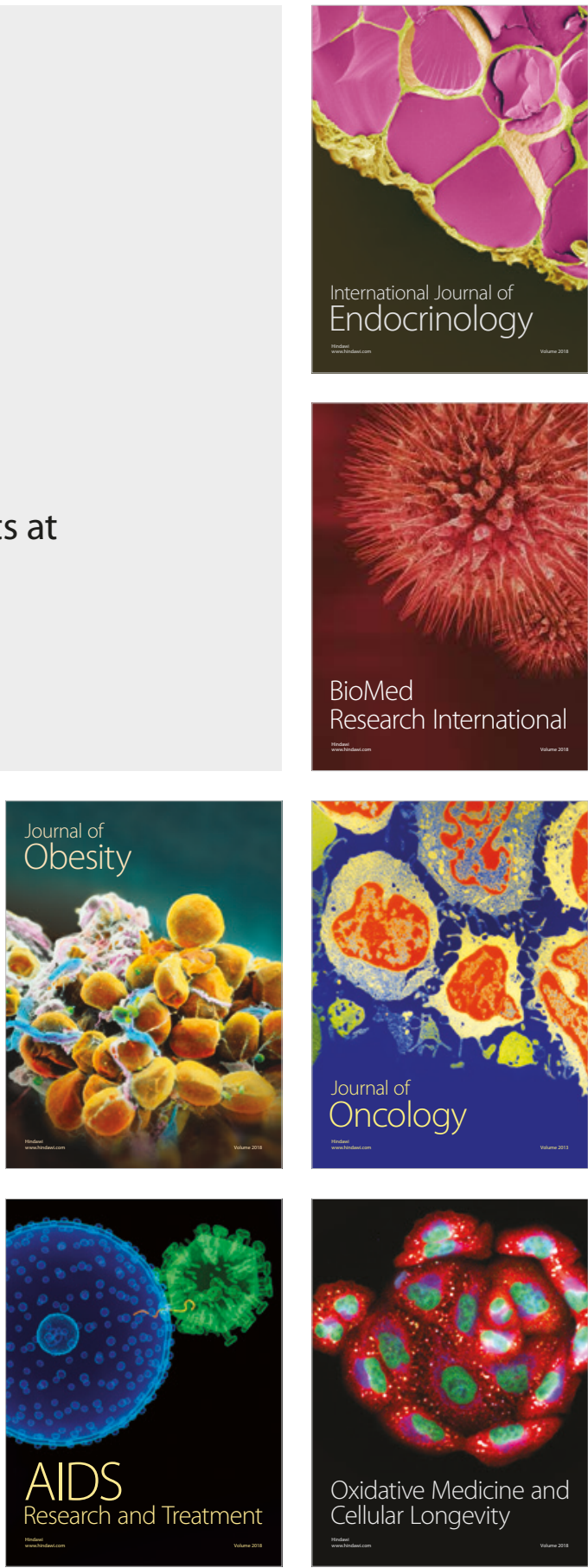